\title{
A reconfigurable damage-tolerant controller based on a modal double-loop framework
}

\author{
Helói F.G. Genari ${ }^{\mathrm{a}, \mathrm{b}, *}$, Nazih Mechbal ${ }^{\mathrm{b}}$, Gérard Coffignal ${ }^{\mathrm{b}}$, \\ Eurípedes G.O. Nóbrega ${ }^{a}$ \\ a Department of Computational Mechanics, University of Campinas, Campinas, Brazil \\ ${ }^{\mathrm{b}}$ Laboratory of Process and Engineering in Mechanics and Materials, Arts et Métiers ParisTech, Paris, France
}

\section{A R T I C L E I N F O}

\section{Article history:}

Received 22 June 2016

Received in revised form

13 November 2016

Accepted 14 November 2016

Available online 7 December 2016

\section{Keywords:}

Damage-tolerant active control

Adaptive modal control

Modal $\mathrm{H}_{\infty}$ control

Modal double-loop control

Active vibration control

\begin{abstract}
A B S T R A C T
Active vibration control of flexible structures has received considerable attention in the latest decades. However, several related control problems remain open to new investigations such as robust performance, spillover instability, and structural changes due to damage. Specifically in the case of damage, it may significantly aggravate closed-loop performance. Damage-tolerant active control is a recent research area that includes structural damage effect reduction in the controller design requirements. This paper presents a novel control method based on a modal double-loop controller design, aiming for vibration reduction of noncollocated flexible structures subject to damage and encompassing online reconfigurability. The first controller is designed for the healthy system in order to comply with predefined performance and robustness requirements, based on modal $\mathrm{H}_{\infty}$ norm. The second controller complements the closed-loop performance if the structure is damaged. A reconfigurable modal technique is adopted to design the second controller, using online modal structural parameter change information to update the controller. To assess the proposed method, finite element models are developed for a case study structure, including health and damage conditions. Results show the effectiveness of the methodology along with performance improvement compared to single-loop controllers based on regular $\mathrm{H}_{\infty}$ and modal $\mathrm{H}_{\infty}$ approaches.
\end{abstract}

\section{Introduction}

Recent research in multifunctional materials and structures has been leading to the innovation of flexible engineering structures, extending their operational goals and safety. Structural and nonstructural functions may now be found in the new generations of flexible structures based on smart materials, which allows the ability of intelligent responses to a variety of stimuli. In particular, structural damage mitigation is prone to take advantage of the smart trend. Current smart structures may embed sensors, actuators, and even digital processors, which can be used to diagnose and to act in order to detect and compensate possible damage effects [1-3]. These structures may indeed present a sophisticated computational level, allowing real-time functions like structural health monitoring (SHM) and active vibration control (AVC). Many techniques have been developed for SHM [4-6] and for AVC [7-9], focusing on damage detection and diagnosis, or adequate

\footnotetext{
* Corresponding author at: Department of Computational Mechanics, University of Campinas, Campinas, Brazil.

E-mail addresses: hegenari@fem.unicamp.br (H.F.G. Genari), nazih.mechbal@ensam.eu (N. Mechbal), gerard.coffignal@ensam.eu (G. Coffignal), egon@fem.unicamp.br (E.G.O. Nóbrega).
} 
performance and robustness for controlled systems. However, SHM and AVC methods are independently designed in general, despite the fact that damage may significantly affect the AVC performance. Aiming to overcome the controller performance decline due to damage, this paper presents a framework to design damage-tolerant active controllers for noncollocated flexible structures, based on a novel double-loop modal controller, which may be adaptively reconfigured in real time. The proposed methodology is inserted into a wide multidisciplinary, involving concepts of AVC, modal control, fault-tolerant control, adaptive control, and SHM, in order to mitigate structural damage effects.

The integration between SHM and vibration control techniques has been receiving considerable attention in recent decades [10-13]. Initially, SHM and control techniques were integrated only in the design phase, with the respective strategy consisting in the simultaneous design of the SHM and control modules [14-16]. These two subsystems were independently operated in the beginning and then, responding to challenges in the civil engineering area, progress has been made in improving the integration approach. New semi-active controllers have been proposed based on magneto-rheological dampers, using the output of the SHM module to update the respective controller parameters [17-20]. However, the increasing demands of larger and lightweight structures with high structural performance lead to the evolution of AVC methods and applications. Despite semi-active controllers intrinsic stability, which represents an advantage for several cases, AVC strategies provide better performance than the passive and the semi-active approaches in structural vibration control [21,22]. After almost two decades of research, the integration of the SHM and the AVC approaches is still a challenge, and regular AVC methods rarely consider, as a design requirement, damage effect mitigation in structural vibrations.

Damage-tolerant active control (DTAC) is a recent research area that has intersections with both fault-tolerant control (FTC) and AVC areas. Specific fault detection and isolation techniques are used by fault-tolerant controllers to provide online parameter adaptation aiming to maintain an adequate performance for controlled systems [23-25]. In the same way, DTAC methods use SHM to design controllers presenting damage-tolerant performance. DTAC methods may be considered an extension of FTC, focusing fundamentally on structural vibration control and aiming to expand the applicability of modern smart structures. However, adapting FTC methods to face damage-induced vibration in flexible structures leads to new challenges. The infinite number of structural vibration modes, which constitutes the beginning of the DTAC research area, demands a controller robustness that is complicated by the dynamic changes due to damage perturbation [3]. The literature regarding vibration control of structures subject to damage is limited. Chattopadhyay et al. [26] used a pole placement technique together with a root mean square method to locate and to mitigate the damage caused by delamination in a composite plate. Umesh and Ganguli [27] investigated the negative velocity feedback control algorithm to compensate the damage effects. Chomette et al. [28] proposed an active modal control for efficient control energy distribution on most damaging modes. Yang et al. [29] used an adaptive controller to reject the vibrations due to earthquakes in a three-story aluminum structure subject to damage. An actuator load was proposed by Ashokkumar [30] to reduce vibration response magnitudes for damage growth mitigation. Ambrosio et al. [31] incorporated the fatigue phenomenon in a cost function, which is then minimized to obtain the controller. Ripamonti et al. [32] investigated an adaptive active vibration control to increase structure life, emphasising the vibration reduction from the most damaging modes. Mechbal and Nóbrega [33] proposed an adaptive methodology based on Lamb wave technique for damage localization and a spatial controller design to reduce vibration energy flow in the damaged region. Recently, the authors submitted papers introducing an $H_{\infty}$ norm applied to the modal control and investigated this methodology applied to damage tolerance [34], considering that the structure distributed stress is known and this permits to infer the most probable regions where damage may occur [35].

Performance and robustness are two important basic requirements for any control design method. Unfortunately, they are opposite to each other, demanding in general a compromise to balance the design requirements. Considering a flexible structure submitted to a regular active vibration controller, performance degradation may be expected in the case of damage occurrence [36]. Regular controllers are not a good solution for structures subject to damage, for instance, considering to ensure safety in an aircraft or in a civil structure. Damage may imply a loss of performance and/or robustness, eventually conducting to instability [37]. An adequate robust active vibration control should be able to automatically accommodate some level of plant model variation, but it cannot protect from structural damage in general. To maintain the overall system stability and an acceptable performance in the case of damage, a specific DTAC design is required [38].

The double-loop robust control concept was proposed by Zhou and Ren [39] to solve the conflict between performance and robustness for the regular feedback framework. The key idea is to use two controllers, in which the first controller is designed to provide performance and the other is designed to guarantee stability and robustness. This control idea has been successfully used as an FTC strategy [40-42]. A new double-loop methodology is presented in this paper, based on the application of the recently introduced modal $H_{\infty}$ norm [34] to control noncollocated flexible structures subject to damage. In this case, the first controller is designed to comply with performance and robustness requirements for the healthy structure. The second controller aims to ensure satisfactory closed-loop performance and robustness including damage effects. To comply with this goal, an original online-reconfigurable technique is adopted to design the second controller, based on a modal state-tracking method to provide the update of the controller parameters. Finite element (FE) models of a case study structure are adopted, considering healthy and increasing damage conditions, in order to examine the proposed double-loop control methodology.

This paper is organized as follows: in Section 2, the adopted DTAC framework is presented; in Section 3, the modal statespace model of a generic flexible structure is introduced; in Section 4, the modal $H_{\infty}$ controller is formulated; in Section 5, the modal double-loop controller is presented; in Section 6, the DTAC double-loop control framework is analyzed, based on simulated results, and final remarks are presented in the last section. 


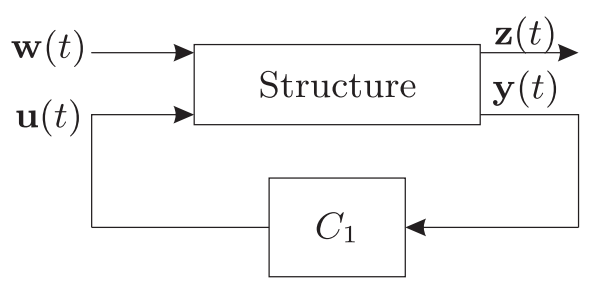

(a) Single loop.

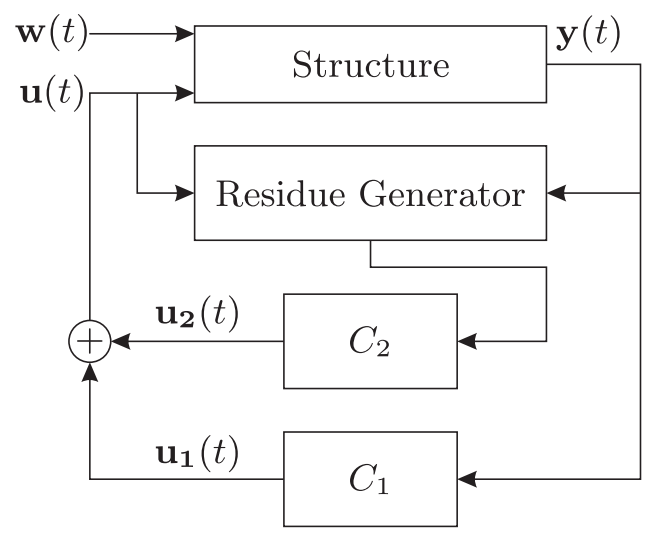

(b) Double loop.

Fig. 1. Standard control strategies.

\section{Adopted DTAC framework}

Regular controllers are designed in order to ensure a specified level of performance and robustness for the closed-loop system. However, both properties are directly influenced by structural model accuracy [43]. Structure dynamics is highly sensitive to damage, which may increase the vibration of both open-loop and closed-loop structures. On the other hand, damage effects over the system dynamics are difficult to predict, hindering to incorporate structural damage information into the controller design. Simply trying to increase the robustness, expecting to accommodate the large model variation due to damage effect, may lead to significant performance loss in the closed-loop system for the healthy structure. Notwithstanding, it does not guarantee acceptable performance if a damage occurs.

To include damage as a control design requirement, the closed-loop system should be able to comply with previously defined performance and robustness requirements for the healthy plant, and also to ensure that, despite the damage dynamic effects, the system maintains an adequate performance level. A methodology for AVC based on the modal doubleloop controller for DTAC applications is here proposed, which satisfies these requirements. Several strategies and approaches are possible for DTAC, similar to existing solutions for FTC. The simple approach is to just design a robust controller in a single loop, as mentioned in the previous paragraph. This strategy is depicted in Fig. 1a, where a robust controller $C_{1}$ is designed to reduce the structural vibration $\mathbf{y}(t)$ caused by the disturbance forces $\mathbf{w}(t)$, satisfying a performance index vector $\mathbf{z}(t)$. However, the unpredictability of the damage effects implies that a good performance is not guaranteed. A better approach is used in [35], in which the key idea is to use previous knowledge acquired from similar structures to predict future damage most likely effects and to design a nonreconfigurable controller to face this probable damage, if and when it happens.

Zhou and Ren [39] proposed the double-loop scheme in Fig. 1b to be used for robustness increase and also for FTC applications. Comparing with the scheme in Fig. $1 \mathrm{a}$, a residue generator and a second controller $C_{2}$ are included. The controller $C_{2}$ acts over the residue signal, which is due to the plant dynamic changes. An application of this configuration is for nonlinear systems, which may be linearized at an operation point, and the second controller compensates any significant variation of this operating point. These two strategies are based on fixed parameter controllers, which is called in the FTC area a passive strategy [44]. However, a more general solution is to design an automatically reconfigurable controller, with the ability to characterize the damage-caused dynamic changes on the plant and adapt the controller online, without the intervention of a human operator. The double-loop scheme is here adopted as a reconfigurable DTAC, considering its simple way to compensate big model changes. In the present case, a linear time-invariant controller is chosen for the first controller, here called the nominal controller, and a reconfigurable controller is adopted as the damage compensator.

Fig. 2 presents the well known FTC reconfigurable framework for a regulator controller [44], which is in general based on a single-loop controller with variable parameters. A residue generator block receives in parallel with the plant both the 


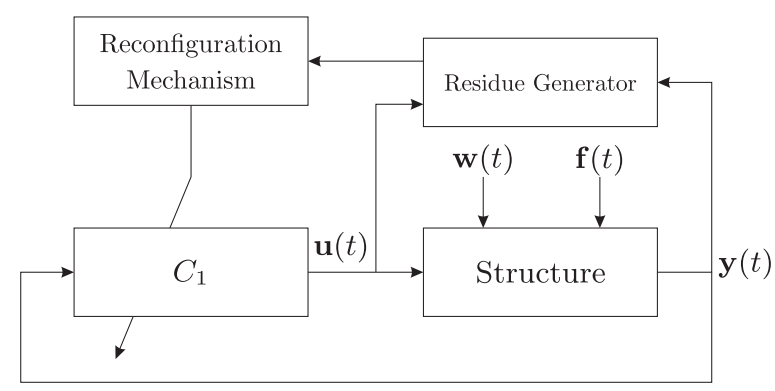

Fig. 2. FTC framework.

control input signal and the real-time measurement output. This block embeds an implicit plant model used to generate the respective residue signal resultant from the processing of these two input signals. Several techniques, known generically as fault detection and isolation (FDI) in the FTC area, may be used to estimate the fault effects, represented by the signal $\mathbf{f}(t)$. In the area of flexible structures, the SHM has similarities in relation to the FDI, with the corresponding differences due to structural behavior particularities. SHM techniques are usually designed to identify, locate, and quantify severity of damage in structures. Considering that smart structures embed transducers, damage may also impact on the transducer behavior, rendering DTAC a more complex problem. However, in both areas, the residue signals need to present the capacity to conduct the reconfiguration of the controller. In order to do this, the reconfiguration mechanism, another functional block that can be seen in Fig. 2, uses the residue to compute online the new controller parameters.

The proposed DTAC framework is presented in Fig. 3. It shows two different controllers, the nominal controller and the damage compensator, which cooperate in order to control the vibration of flexible structures subject to damage. The nominal controller parameters are set in the design phase to provide the disturbance rejection and they are not updated. The damage compensator parameters are reconfigured online to face the damage consequences on the plant behavior. The modal $H_{\infty}$ approach is a recently developed robust control technique [34] and is here adopted to design the nominal controller. The modal approach provides high authority over the control energy distribution among the modes, reflecting a designer-chosen compromise to guarantee a very good performance for the healthy structure. As such, this healthy closedloop model is also adopted as the reference to generate the desired performance output. This means that the damage compensator goal is to maintain the same performance that was present in the plant before the damage occurrence. The damage effects are assumed as a bounded unknown input signal $\varphi(t)$. To design the reconfigurable damage compensator, a state-tracking strategy is here modified, based on a model reference adaptive control approach [45], which includes the modal behavior as a design requirement. The estimated state vector is compared to the reference state vector, generating a residue vector which the reconfiguration mechanism uses to adapt the damage compensator parameters. In the next sections, the proposed DTAC methodology is detailed, in order to explain how the double-loop approach may be used to implement a reconfigurable controller.

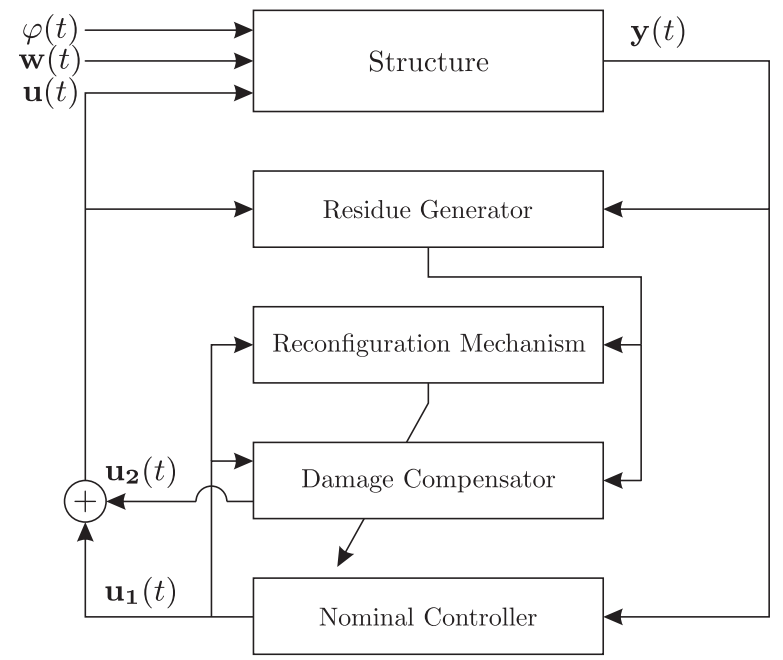

Fig. 3. Proposed DTAC framework. 


\section{Flexible structure model}

The second-order differential matrix equation is commonly used in the analysis of structural dynamics and it can be easily converted into the modal state-space representation used in control applications. For the sake of completeness, this section presents a brief overview of the modal state-space representation to describe the structure behavior, adopting a modal representation according to the proposed controller design method.

A generic flexible structure in nodal coordinates with multiple transducers can be described by the following pair of equations:

$$
\begin{aligned}
& \mathbf{M} \ddot{\mathbf{p}}(t)+\mathbf{D} \dot{\mathbf{p}}(t)+\mathbf{K} \mathbf{p}(t)=\mathbf{B}_{\mathbf{w}} \mathbf{w}(t)+\mathbf{B}_{\mathbf{u}} \mathbf{u}(t) \\
& \mathbf{y}(t)=\mathbf{C}_{\mathbf{d}} \mathbf{p}(t)+\mathbf{C}_{\mathbf{v}} \dot{\mathbf{p}}(t)+\mathbf{C}_{\mathbf{w}} \mathbf{w}(t),
\end{aligned}
$$

in which $\mathbf{p}(t)$ denotes the displacement vector, $\mathbf{M}$ is the mass matrix, $\mathbf{D}$ is the damping matrix, $\mathbf{K}$ is the stiffness matrix, $\mathbf{B}_{\mathbf{w}}$ and $\mathbf{B}_{\mathbf{u}}$ are the respective input matrices, where $\mathbf{w}(t)$ are the disturbance forces acting on the structure, $\mathbf{u}(t)$ are the control forces, and $\mathbf{y}(t)$ are the measured output signals, modeled through the output matrices $\mathbf{C}_{\mathbf{d}}, \mathbf{C}_{\mathbf{v}}$, and $\mathbf{C}_{\mathbf{w}}$. Usually, the structure FE models have a very large order, however, considering that there exists a frequency band of interest, a proper number of modes are adopted to choose a manageable model. The following modal matrix is used to decouple the modal equations, considering $m$ as the adopted number of modes:

$$
\Phi=\left[\begin{array}{llll}
\phi_{1} & \phi_{2} & \ldots & \phi_{\mathrm{m}}
\end{array}\right],
$$

where $\phi_{\mathrm{i}}$ is the $i$ mode shape.

A transformation into modal coordinates is obtained using $\mathbf{p}(t)=\mathbf{\Phi q}(t)$. Pre-multiplying Eq. (1) by $\boldsymbol{\Phi}^{T}$ gives:

$$
\begin{aligned}
& \boldsymbol{\Phi}^{T} \mathbf{M} \Phi \ddot{\mathbf{q}}(t)+\boldsymbol{\Phi}^{T} \mathbf{D} \Phi \dot{\mathbf{q}}(t)+\boldsymbol{\Phi}^{T} \mathbf{K} \Phi \mathbf{q}(t)=\boldsymbol{\Phi}^{T} \mathbf{B}_{\mathbf{w}} \mathbf{w}(t)+\boldsymbol{\Phi}^{T} \mathbf{B}_{\mathbf{u}} \mathbf{u}(t) \\
& \mathbf{y}(t)=\mathbf{C}_{\mathbf{d}} \Phi \mathbf{q}(t)+\mathbf{C}_{\mathbf{v}} \Phi \dot{\mathbf{q}}(t)+\mathbf{C}_{\mathbf{w}} \mathbf{w}(t),
\end{aligned}
$$

which may be written as:

$$
\begin{aligned}
& \ddot{\mathbf{q}}(t)+\mathbf{M}_{\mathbf{m}}^{-1} \mathbf{D}_{\mathbf{m}} \dot{\mathbf{q}}(t)+\mathbf{M}_{\mathbf{m}}^{-1} \mathbf{K}_{\mathbf{m}} \mathbf{q}(t)=\mathbf{M}_{\mathbf{m}}^{-1} \mathbf{B}_{\mathbf{w}_{\mathbf{m}}} \mathbf{w}(t)+\mathbf{M}_{\mathbf{m}}^{-1} \mathbf{B}_{\mathbf{u}_{\mathbf{m}}} \mathbf{u}(t) \\
& \mathbf{y}(t)=\mathbf{C}_{\mathbf{d}_{\mathbf{m}}} \mathbf{q}(t)+\mathbf{C}_{\mathbf{v}_{\mathbf{m}}} \dot{\mathbf{q}}(t)+\mathbf{C}_{\mathbf{w}} \mathbf{w}(t),
\end{aligned}
$$

where $\mathbf{M}_{\mathrm{m}}=\boldsymbol{\Phi}^{T} \mathbf{M} \boldsymbol{\Phi}$ is assumed nonsingular, $\mathbf{D}_{\mathrm{m}}=\boldsymbol{\Phi}^{T} \mathbf{D} \boldsymbol{\Phi}, \mathbf{K}_{\mathrm{m}}=\boldsymbol{\Phi}^{T} \mathbf{K} \boldsymbol{\Phi}, \mathbf{B}_{\mathbf{w}_{\mathrm{m}}}=\boldsymbol{\Phi}^{T} \mathbf{B}_{\mathbf{w}}, \mathbf{B}_{\mathbf{u}_{\mathrm{m}}}=\boldsymbol{\Phi}^{T} \mathbf{B}_{\mathbf{u}}, \mathbf{C}_{\mathbf{d}_{\mathrm{m}}}=\mathbf{C}_{\mathrm{d}} \boldsymbol{\Phi}$, and $\mathbf{C}_{\mathbf{v}_{\mathrm{m}}}=\mathbf{C}_{\mathrm{v}} \boldsymbol{\Phi}$. Matrices $\mathbf{M}_{\mathbf{m}}$ and $\mathbf{K}_{\mathbf{m}}$ are diagonal while $\mathbf{D}_{\mathbf{m}}$ is not necessarily diagonal. In order to make the modal matrix also diagonal, a linear combination of the stiffness and mass matrices is usually adopted: $\mathbf{D}=\alpha \mathbf{M}+\beta \mathbf{K}$ for $\alpha, \beta \geq 0$. This is a reasonable and usual assumption, considering that flexible structures have small damping factors.

From Eqs. (2) and (3), the second-order differential equations can be transformed into the following state-space representation:

$$
\begin{aligned}
\dot{\mathbf{x}}(t) & =\mathbf{A x}(t)+\mathbf{B}_{1} \mathbf{w}(t)+\mathbf{B}_{\mathbf{2}} \mathbf{u}(t) \\
\mathbf{y}(t) & =\mathbf{C}_{\mathbf{2}} \mathbf{x}(t)+\mathbf{D}_{\mathbf{2 1}} \mathbf{w}(t),
\end{aligned}
$$

in which $\mathbf{x}(t)$ is the state vector. The state-variable definition conducts to a specific canonical model, of which some examples are given in [46]. For this paper, the following state-space model structure is adopted:

$$
A=\left[\begin{array}{cccc}
\mathbf{A}_{1} & \mathbf{0} & \cdots & \mathbf{0} \\
\mathbf{0} & \mathbf{A}_{2} & \cdots & \mathbf{0} \\
\vdots & \vdots & \ddots & \vdots \\
\mathbf{0} & \mathbf{0} & \cdots & \mathbf{A}_{\mathbf{m}}
\end{array}\right], \mathbf{B}_{\mathbf{1}}=\left[\begin{array}{c}
\mathbf{B}_{11} \\
\mathbf{B}_{12} \\
\vdots \\
\mathbf{B}_{1 \mathrm{~m}}
\end{array}\right], \mathbf{B}_{\mathbf{2}}=\left[\begin{array}{c}
\mathbf{B}_{21} \\
\mathbf{B}_{22} \\
\vdots \\
\mathbf{B}_{2 \mathrm{~m}}
\end{array}\right], \quad \text { and } \quad \mathbf{C}_{\mathbf{2}}=\left[\begin{array}{c}
\mathbf{C}_{21}^{T} \\
\mathbf{C}_{22}^{T} \\
\vdots \\
\mathbf{C}_{2 \mathrm{~m}}^{T}
\end{array}\right]^{T} \text {, }
$$

where $\mathbf{A}_{i}$ is a $2 \times 2$ matrix for $i=1 \ldots m$, therefore it isolates each mode.

\section{Nominal controller}

The plant to be controlled is given by Eq. (5), in which a performance indicator is introduced as an output vector $\mathbf{z}(t)$, considering the modal canonical representation of the previous section. In general, this performance output index depends only on time. However, to include modal requirements, it is necessary to transform the regular performance output. Considering only the time-dependent performance vector, the following state-space equations are used to design the regular robust controllers: 


$$
\begin{gathered}
\dot{\mathbf{x}}(t)=\mathbf{A x}(t)+\mathbf{B}_{\mathbf{1}} \mathbf{w}(t)+\mathbf{B}_{\mathbf{2}} \mathbf{u}(t) \\
\mathbf{z}(t)=\mathbf{C}_{\mathbf{1}} \mathbf{x}(t)+\mathbf{D}_{\mathbf{1 1}} \mathbf{W}(t)+\mathbf{D}_{\mathbf{1 2}} \mathbf{u}(t) \\
\mathbf{y}(t)=\mathbf{C}_{\mathbf{2}} \mathbf{x}(t)+\mathbf{D}_{\mathbf{2 1}} \mathbf{w}(t)
\end{gathered}
$$

in which matrices $\mathbf{C}_{\mathbf{1}}, \mathbf{D}_{\mathbf{1 1}}$, and $\mathbf{D}_{\mathbf{1 2}}$ are chosen to define the desired performance vector $\mathbf{z}(t)$. In addition, all vectors and matrices have appropriate dimensions related to the number of inputs and outputs and to the model order. Assuming the modal model, the respective performance vector can be described in terms of modal performances as $\mathbf{z}(t)=\sum_{i=1}^{m} \mathbf{z}_{i}(t)$, in which $\mathbf{z}_{i}(t)$ represents the performance signals relative to mode $i$. Moreover, the disturbance vector $\mathbf{w}(t)$, with $\left.\mathbf{w} \in \mathcal{L}_{2}\right] 0$, $\infty$, can be filtered into $m$ contiguous frequency bands such that $\mathbf{w}(t)=\sum_{i=1}^{m} \mathbf{w}_{i}(t)$, in which each band is chosen in order to contain only one distinct mode.

Given a state-space controller $K_{c}$ as:

$$
\begin{aligned}
& \dot{\mathbf{x}}_{c}(t)=\mathbf{A}_{c} \mathbf{x}_{c}(t)+\mathbf{B}_{c} \mathbf{y}(t) \\
& \mathbf{u}(t)=\mathbf{C}_{c} \mathbf{x}_{c}(t)+\mathbf{D}_{c} \mathbf{y}(t),
\end{aligned}
$$

the regular $H_{\infty}$ control problem is to find the controller matrices by the minimization of the following objective function:

$$
J_{\infty}=\frac{\int_{0}^{\infty} \mathbf{z}^{T}(t) \mathbf{z}(t) d t}{\int_{0}^{\infty} \mathbf{w}^{T}(t) \mathbf{w}(t) d t}
$$

A modal $H_{\infty}$ norm, which is dependent of the modal weighing matrices, is introduced and minimized to find the modal $H_{\infty}$ controller, according to Eq. (6), such that the closed-loop system satisfies:

$$
\underbrace{\sup }_{K_{c} \in V_{\left.\mathbf{w} \in \mathcal{L}_{2}\right] 0, \infty[}^{\inf }} J_{m}<\gamma^{2}
$$

in which $V$ represents the set of all controllers that stabilize the plant and:

$$
J_{m}=\frac{\sum_{i=1}^{m} \int_{0}^{\infty} \mathbf{z}_{i}^{T}(t) \mathbf{Q}_{i} \mathbf{z}_{i}(t) d t}{\sum_{i=1}^{m} \int_{0}^{\infty} \mathbf{w}_{i}^{T}(t) \mathbf{w}_{i}(t) d t}
$$

where the diagonal matrix $\mathbf{Q}_{i}>0$ weights the mode $i$, whose results conduct to the sought mode selectivity.

Defining a new modal performance indicator as:

$$
\mathbf{z}_{\mathbf{p}}(t)=\boldsymbol{\Gamma} \mathbf{x}(t)+\Theta \mathbf{w}(t)+\Lambda \mathbf{u}(t)
$$

with:

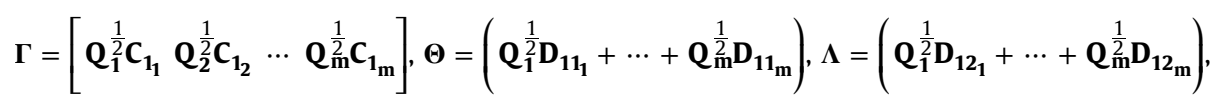

where $\mathbf{C}_{\mathbf{1}}, \mathbf{D}_{\mathbf{1 1}_{i}}$, and $\mathbf{D}_{\mathbf{1 2}_{i}}$ correspond to the respective mode $i$ submatrices in $\mathbf{C}_{\mathbf{1}}, \mathbf{D}_{\mathbf{1 1}}$, and $\mathbf{D}_{\mathbf{1 2}}$. The modal problem given in Eq. (7) can be equivalently solved using the following objective function [34]:

$$
J_{\infty}=\frac{\int_{0}^{\infty} \mathbf{z}_{\mathbf{p}}^{T}(t) \mathbf{z}_{\mathbf{p}}(t) d t}{\int_{0}^{\infty} \mathbf{w}^{T}(t) \mathbf{w}(t) d t},
$$

meaning that known tools may be applied to solve this problem.

Fig. 4 represents a general MIMO system framework adopted to design the modal active controller, including weighing filters for frequency band restrictions to avoid spillover, to limit the control signals, and to balance the relation between the

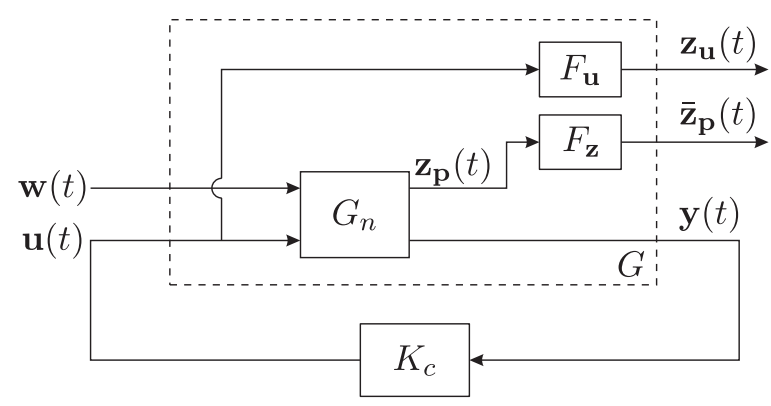

Fig. 4. Block diagram of the $H_{\infty}$ control problem. 
disturbances and the performance index. This is done through the filters $F_{\mathbf{u}}$ and $F_{\mathbf{z}}$ whose outputs are respectively $\mathbf{z}_{\mathbf{u}}(t)$ and $\overline{\mathbf{z}}_{\mathbf{p}}(t)$, which define the generalized performance vector as $\hat{\mathbf{z}}(t)=\left[\overline{\mathbf{z}}_{\mathbf{p}}^{T}(t) \mathbf{z}_{\mathbf{u}}^{\mathrm{T}}(t)\right]^{T}$. The nominal plant $G_{n}$ is obtained from the modal reduced model, $G$ is the generalized plant, including the filters, and the respective nominal controller is $K_{c}$.

To choose the appropriate weighing filters represents an important step in the controller design process, which may involve several iterations to achieve fine tuning. Usually, $F_{\mathbf{z}}(s)$ is chosen as a low-pass filter and $F_{\mathbf{u}}(s)$ is designed as a highpass filter, designed according to the following equations [47]:

$$
F_{\mathbf{z}}(s)=\left(\frac{\frac{s}{\sqrt[k]{M}}+\omega_{c}}{s+\omega_{c} \sqrt[k]{\varepsilon}}\right)^{k} \text { and } F_{\mathbf{u}}(s)=\left(\frac{s+\frac{\omega_{c}}{\sqrt[k]{M}}}{s \sqrt[k]{\varepsilon}+\omega_{c}}\right)^{k}
$$

in which $\omega_{\mathrm{c}}, k, \varepsilon$, and $M$ determine the transition frequency between rejection band and passband, the filter order, the gain at pass band, and the gain at rejection band, respectively.

The transfer matrix between the performance output $\hat{\mathbf{z}}(t)$ and the disturbance $\mathbf{w}(t)$ is defined as $T_{\hat{\mathbf{z}} \mathbf{w}}$, where $\left\|T_{\hat{\mathbf{z}} \mathbf{w}}\right\|_{\infty}$ represents the $H_{\infty}$ norm of $G$. The modal $H_{\infty}$ controller design consists in finding a central controller $K_{c}$ that minimizes $\left\|T_{\hat{\mathbf{z}} \mathbf{w}}\right\|_{\infty}$. Considering the controller represented by Eq. (6) and the generalized plant $G$ with their filters, then the closed-loop system is given by [47]:

$$
\begin{array}{r}
\dot{\mathbf{\mathbf { x }}}(t)=\tilde{\mathbf{A}} \tilde{\mathbf{x}}(t)+\tilde{\mathbf{B}} \mathbf{w}(t) \\
\hat{\mathbf{z}}(t)=\tilde{\mathbf{C}} \tilde{\mathbf{x}}(t)+\tilde{\mathbf{D}} \mathbf{w}(t) .
\end{array}
$$

Based on the bounded real lemma [48], $\tilde{A}$ is stable and $\left\|T_{\hat{\mathbf{z}} \mathbf{w}}\right\|_{\infty}<\rho$ if and only if there exists a symmetric matrix $\tilde{\mathbf{P}}$ that satisfies the following linear matrix inequalities (LMIs) [49]:

$$
\left[\begin{array}{ccc}
\tilde{\mathbf{A}}^{T} \tilde{\mathbf{P}}+\tilde{\mathbf{P}} \tilde{\mathbf{A}} & \tilde{\mathbf{P}} \tilde{\mathbf{B}} & \tilde{\mathbf{C}}^{T} \\
\tilde{\mathbf{B}}^{T} \tilde{\mathbf{P}} & -\rho \mathbf{I} & \tilde{\mathbf{D}}^{T} \\
\tilde{\mathbf{C}} & \tilde{\mathbf{D}} & -\rho \mathbf{I}
\end{array}\right]<0, \tilde{\mathbf{P}}>0,
$$

in which it is usual to adopt a suboptimal problem to solve the respective $H_{\infty}$ control problem, based on successive iterations to find the controller matrices. In order to find $\left\|T_{\hat{\mathbf{z}} \mathbf{w}}\right\|_{\infty} \leq \rho$, the convex optimization problem can be adopted and solved by well-known procedures, see [50] for more details.

\section{DTAC double-loop reconfigurable controller framework}

Fig. 5 shows the detailed block diagram of the DTAC framework, including the plant and five constitutive modules, respectively the nominal controller, the modal observer, the reference model, the reconfiguration mechanism, and the damage compensator. The nominal controller generates the control signal $\mathbf{u}_{1}(t)$ and is already described in the previous section. The damage compensator generates the control signal $\mathbf{u}_{2}(t)$, based on the updated parameters provided by the reconfiguration mechanism. An SHM module encompasses a modal state observer and a reference model block, whose

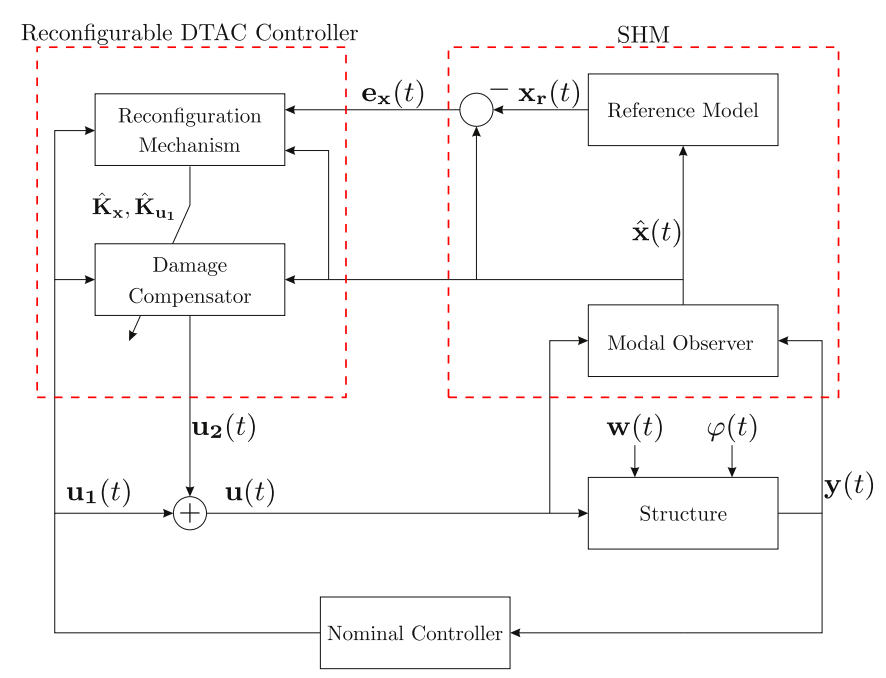

Fig. 5. Detailed block diagram of the DTAC framework. 
output $\mathbf{x}_{\mathbf{r}}(t)$ is compared to the observed state $\hat{\mathbf{x}}(t)$ to generate the residue $\mathbf{e}_{\mathbf{x}}(t)=\hat{\mathbf{x}}(t)-\mathbf{x}_{\mathbf{r}}(t)$. The residue is essential for the parameter reconfiguration. These modules are detailed in the next subsections.

\subsection{Modal observer design}

The modal state vector $\mathbf{x}(t)$ is estimated online based on the measured output signal $\mathbf{y}(t)$ and the control signal $\mathbf{u}(t)$, which contain the respective damage information. The following representation is adopted to include damage in the statespace model:

$$
\begin{aligned}
& \dot{\mathbf{x}}(t)=\mathbf{A} \mathbf{x}(t)+\mathbf{B}_{\mathbf{1}} \mathbf{w}(t)+\mathbf{B}_{\mathbf{2}} \mathbf{u}(t)+\mathbf{B}_{\mathbf{3}} \varphi(t) \\
& \mathbf{y}(t)=\mathbf{C}_{\mathbf{2}} \mathbf{x}(t)+\mathbf{D}_{\mathbf{2 1}} \mathbf{w}(t),
\end{aligned}
$$

where the pair $\left(\mathbf{A}, \mathbf{C}_{\mathbf{2}}\right.$ ) is assumed observable, $\varphi \in \mathcal{L}_{2}$ represents the unknown damage signal, and $\mathbf{B}_{\mathbf{3}}$ is responsible for balancing the damage in each mode. Considering that the controller objective is to compensate the modal vibration in terms of the modal $H_{\infty}$ norm and respective assumed weights, the control transducer position implies that it guarantees the authority to do so. Therefore, it is possible to admit that $\mathbf{B}_{\mathbf{3}}=\mathbf{B}_{\mathbf{2}}$, without loss of generality.

The following traditional Luenberger observer is adopted to estimate the modal state vector:

$$
\begin{aligned}
\dot{\hat{\mathbf{x}}}(t) & =\mathbf{A} \hat{\mathbf{x}}(t)+\mathbf{B}_{\mathbf{2}} \mathbf{u}(t)+\mathbf{L}(\mathbf{y}(t)-\hat{\mathbf{y}}(t)) \\
\hat{\mathbf{y}}(t) & =\mathbf{C}_{\mathbf{2}} \hat{\mathbf{x}}(t),
\end{aligned}
$$

in which $\hat{\mathbf{x}}(t)$ and $\hat{\mathbf{y}}(t)$ are the estimations of the state and the output vectors. The gain $\mathbf{L}$ is chosen so that $\hat{\mathbf{x}}(t)$ converges to $\mathbf{x}(t)$ asymptotically.

The estimation error dynamics $\mathbf{e}(t)$ is given by:

$$
\dot{\mathbf{e}}(t)=\dot{\mathbf{x}}(t)-\dot{\hat{\mathbf{x}}}(t)=\mathbf{A} \mathbf{x}(t)+\mathbf{B}_{1} \mathbf{w}(t)+\mathbf{B}_{2} \mathbf{u}(t)-\mathbf{A} \hat{\mathbf{x}}(t)-\mathbf{B}_{2} \mathbf{u}(t)-\mathbf{L}\left(\mathbf{y}(t)-\mathbf{C}_{2} \hat{\mathbf{x}}(t)\right)+\mathbf{B}_{2} \boldsymbol{\varphi}(\mathbf{t})=\mathbf{N e}(t)+\mathbf{E w}(t)+\mathbf{B}_{2} \varphi(t),
$$

in which $\mathbf{N}=\mathbf{A}-\mathbf{L C}_{\mathbf{2}}$ and $\mathbf{E}=\mathbf{B}_{\mathbf{1}}-\mathbf{L D}_{\mathbf{2 1}}$.

The observer gain $\mathbf{L}$ is computed in order to ensure stability of the estimation error dynamics and to attenuate the influence of damage and disturbance in the state estimation. For this purpose, the following theorem is proposed to give an observer solution based on an LMI approach.

Theorem 1. The estimation error dynamics given in Eq. (11) is globally stable in relation to the $H_{\infty}$ performance if there exist matrices $\overline{\mathbf{P}}=\overline{\mathbf{P}}^{T} \geq 0, \Theta$, and scalars $\gamma, \nu, \alpha>0$ that satisfy $\|\mathbf{e}(t)\|_{2} \leq \gamma\|\boldsymbol{\varphi}(t)\|_{2},\|\mathbf{w}(t)\|_{2} \leq \nu$, and the following LMI:

$$
\left[\begin{array}{ccc}
\mathbf{A}^{T} \overline{\mathbf{P}}-\mathbf{C}_{\mathbf{2}}^{T} \boldsymbol{\Theta}^{T}+\overline{\mathbf{P}} \mathbf{A}-\mathbf{\Theta C}_{\mathbf{2}}+I+\alpha \mathbf{I} & \overline{\mathbf{P}}_{\mathbf{2}} & \overline{\mathbf{P B}}_{\mathbf{1}}-\mathbf{\Theta D}_{\mathbf{2 1}} \\
\mathbf{B}_{\mathbf{2}}^{T} \overline{\mathbf{P}} & -\mu \mathbf{I} & \mathbf{0} \\
\mathbf{B}_{\mathbf{1}}^{T} \overline{\mathbf{P}}-\mathbf{D}_{\mathbf{2} 1}^{T} \boldsymbol{\Theta}^{T} & \mathbf{0} & -\frac{1}{\beta} \mathbf{I}
\end{array}\right]<0,
$$

in which $\mu=\gamma^{2}, \beta=\alpha^{-1} \nu^{2}$, and the observer gain is computed as $\mathbf{L}=\overline{\mathbf{P}}^{-1} \mathbf{\Theta}$.

Proof. The $\mathcal{L}_{2}$-induced norm from $\varphi(t)$ to $\mathbf{e}(t)$ considering $\gamma>0$ is given by:

$$
\int_{0}^{\infty} \mathbf{e}^{T}(t) \mathbf{e}(t) \mathrm{d} t<\gamma^{2} \int_{0}^{\infty} \varphi^{T}(t) \varphi(t) \mathrm{d} t
$$

The performance index $J_{\varphi_{e}}$ is defined from the inequality (13) as:

$$
J_{\varphi_{\mathbf{e}}}=\int_{0}^{\infty}\left\{\mathbf{e}^{T}(t) \mathbf{e}(t)-\gamma^{2} \boldsymbol{\varphi}^{T}(t) \boldsymbol{\varphi}(t)\right\} \mathrm{d} t
$$

Considering the Lyapunov candidate function $V(\mathbf{e}(t))$ and that the boundary conditions are null, i.e., $\left.V(\mathbf{e}(t))\right|_{t=0}=0$ and $\left.V(\mathbf{e}(t))\right|_{t \rightarrow \infty} \rightarrow 0$, then:

$$
\begin{aligned}
J_{\varphi_{\mathbf{e}}} & =\int_{0}^{\infty}\left\{\mathbf{e}^{T}(t) \mathbf{e}(t)-\gamma^{2} \boldsymbol{\varphi}^{T}(t) \boldsymbol{\varphi}(t)+\dot{V}(\mathbf{e}(t))-\dot{V}(\mathbf{e}(t))\right\} \mathrm{d} t \\
& =\int_{0}^{\infty}\left\{\mathbf{e}^{T}(t) \mathbf{e}(t)-\gamma^{2} \boldsymbol{\varphi}^{T}(t) \boldsymbol{\varphi}(t)+\dot{V}(\mathbf{e}(t))\right\} \mathrm{d} t+\left.V(\mathbf{e}(t))\right|_{t=0}-\left.V(\mathbf{e}(t))\right|_{t \rightarrow \infty} \\
& =\int_{0}^{\infty}\left\{\mathbf{e}^{T}(t) \mathbf{e}(t)-\gamma^{2} \boldsymbol{\varphi}^{T}(t) \boldsymbol{\varphi}(t)+\dot{V}(\mathbf{e}(t))\right\} \mathrm{d} t .
\end{aligned}
$$

The Lyapunov quadratic function is chosen as $V(\mathbf{e}(t))=\mathbf{e}^{T}(t) \overline{\mathbf{P}} \mathbf{e}(t)$. Thus, the derivative of $V(\mathbf{e}(t))$ along Eq. (11) is given by:

$$
\dot{V}(\mathbf{e}(t))=\dot{\mathbf{e}}^{T}(t) \overline{\mathbf{P}} \mathbf{e}(t)+\mathbf{e}^{T}(t) \overline{\mathbf{P}} \dot{\mathbf{e}}(t)=\mathbf{e}^{T}(t)\left(\mathbf{N}^{T} \overline{\mathbf{P}}+\overline{\mathbf{P}} \mathbf{N}\right) \mathbf{e}(t)+2 \mathbf{e}^{T}(t) \overline{\mathbf{P}} \mathbf{E w}(t)+\boldsymbol{\varphi}^{T}(t) \mathbf{B}_{2}^{T} \overline{\mathbf{P}}(t)+\mathbf{e}^{T}(t) \overline{\mathbf{P}} \mathbf{B}_{2} \boldsymbol{\varphi}(t) .
$$

For $\|\mathbf{w}(t)\|_{2} \leq \nu$, it follows that: 


$$
\dot{V}(\mathbf{e}(t))<\mathbf{e}^{T}(t)\left(\mathbf{N}^{T} \overline{\mathbf{P}}+\overline{\mathbf{P}} \mathbf{N}\right) \mathbf{e}(t)+2 \nu\left\|\mathbf{e}^{T}(t) \overline{\mathbf{P}} \mathbf{E}\right\|_{2}+\boldsymbol{\varphi}^{T}(t) \mathbf{B}_{2}^{T} \overline{\mathbf{P}}(t)+\mathbf{e}^{T}(t) \overline{\mathbf{P}} \mathbf{B}_{2} \boldsymbol{\varphi}(t) .
$$

Considering a scalar $\alpha>0$, the following expression is verified ${ }^{1}$ :

$$
2 \nu\left\|\mathbf{e}^{T}(t) \overline{\mathbf{P}} \mathbf{E}\right\|_{2} \leq \alpha^{-1} \nu^{2}\left\|\mathbf{e}^{T}(t) \overline{\mathbf{P}} \mathbf{E}\right\|_{2}^{2}+\alpha,
$$

which leads to:

$$
\dot{V}(\mathbf{e}(t))<\mathbf{e}^{T}(t)\left(\mathbf{N}^{T} \overline{\mathbf{P}}+\overline{\mathbf{P}} \mathbf{N}\right) \mathbf{e}(t)+\beta\left\|\mathbf{e}^{T}(t) \overline{\mathbf{P}} \mathbf{E}\right\|_{2}^{2}+\alpha+\boldsymbol{\varphi}^{T}(t) \mathbf{B}_{2}^{T} \overline{\mathbf{P}}(t)+\mathbf{e}^{T}(t) \overline{\mathbf{P}} \mathbf{B}_{2} \varphi(t),
$$

where $\beta=\alpha^{-1} \nu^{2}$.

Substituting inequality (15) into Eq. (14), the performance index is then given by [51]:

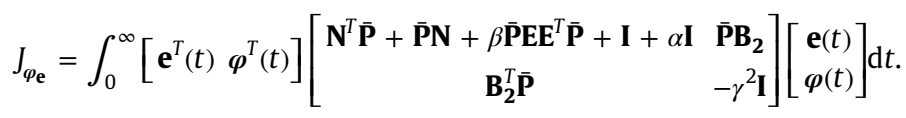

The condition to satisfy the inequality (13) is $J_{\varphi_{\mathrm{e}}}<0$, leading to:

$$
\left[\begin{array}{cc}
\mathbf{N}^{T} \overline{\mathbf{P}}+\overline{\mathbf{P}} \mathbf{N}+\beta \overline{\mathbf{P}} \mathbf{E}^{T} \overline{\mathbf{P}}+\mathbf{I}+\alpha \mathbf{I} & \overline{\mathbf{P}} \mathbf{B}_{\mathbf{2}} \\
\mathbf{B}_{2}^{T} \overline{\mathbf{P}} & -\gamma^{2} \mathbf{I}
\end{array}\right]<0 .
$$

Considering the Schur complement, the inequality (16) becomes:

$$
\left[\begin{array}{ccc}
\mathbf{N}^{T} \overline{\mathbf{P}}+\overline{\mathbf{P}} \mathbf{N}+\mathbf{I}+\alpha \mathbf{I} & \overline{\mathbf{P}} \mathbf{B}_{2} & \overline{\mathbf{P}} \mathbf{E} \\
\mathbf{B}_{2}^{T} \overline{\mathbf{P}} & -\gamma^{2} \mathbf{I} & \mathbf{0} \\
\mathbf{E}^{T} \overline{\mathbf{P}} & \mathbf{0} & -\frac{1}{\beta} \mathbf{I}
\end{array}\right]<0 .
$$

A new matrix $\mathbf{\Theta}=\overline{\mathbf{P}} \mathbf{L}$ together with $\mathbf{N}=\mathbf{A}-\mathbf{L} \mathbf{C}_{\mathbf{2}}, \mathbf{E}=\mathbf{B}_{\mathbf{1}}-\mathbf{L D}_{\mathbf{2 1}}$, and $\mu=\gamma^{2}$ is used in order to eliminate the quadratic terms of the inequality (17):

$$
\left[\begin{array}{lcc}
\mathbf{A}^{T} \overline{\mathbf{P}}-\mathbf{C}_{\mathbf{2}}^{T} \boldsymbol{\Theta}^{T}+\overline{\mathbf{P}} \mathbf{A}-\boldsymbol{\Theta} \mathbf{C}_{\mathbf{2}}+\mathbf{I}+\alpha \mathbf{I} & \overline{\mathbf{P}} \mathbf{B}_{\mathbf{2}} & \overline{\mathbf{P}} \mathbf{B}_{\mathbf{1}}-\boldsymbol{\Theta} \mathbf{D}_{\mathbf{2 1}} \\
\mathbf{B}_{\mathbf{2}}^{T} \overline{\mathbf{P}} & -\mu \mathbf{I} & \mathbf{0} \\
\mathbf{B}_{\mathbf{1}}^{T} \overline{\mathbf{P}}-\mathbf{D}_{2 \mathbf{1}}^{T} \boldsymbol{\Theta}^{T} & \mathbf{0} & -\frac{1}{\beta} \mathbf{I}
\end{array}\right]<0,
$$

where $\mu, \overline{\mathbf{P}}$, and $\boldsymbol{\Theta}$ are the variables to be computed via the numerical solution of LMI. This completes the proof.

The proposed modal observer is based on the reduced model order. However, the sensor outputs may be affected by residual modes through the observation spillover [52], which may introduce an additive noise in the state-vector estimation. To avoid this effect, the state pair of each mode is filtered using a bandpass filter focused on the respective resonant frequency.

\subsection{Reference model}

As already mentioned, the reference model uses the closed loop of the healthy structure model given in Eq. (5) under the nominal controller represented in Eq. (6). The reference model module continuously generates the desired vector state $x_{r}(t)$, i.e., without the damage influence. For this purpose, the disturbance input should be known in order to be used to excite the reference model. Usually, it is difficult to estimate online the disturbance forces acting on practical structures. To overcome this limitation, an estimation of the worst-case disturbance is used instead, according to the following equation:

$$
\mathbf{w}^{*}(t)=\left(\rho^{2} \mathbf{I}-\tilde{\mathbf{D}}^{T} \tilde{\mathbf{D}}\right)^{-1}\left(\tilde{\mathbf{B}}^{T} \tilde{\mathbf{P}}+\tilde{\mathbf{D}}^{T} \tilde{\mathbf{C}}\right) \hat{\mathbf{x}}(t),
$$

in which this equation is based on the solution of the nominal controller problem, represented here by the set of inequalities (10) (see [53] for more details). Notice that using the worst-case disturbance, the estimated state vector is a conservative choice, always guaranteeing the highest possible residue. Finally, it worths to mention that a plant model with additional high-order modes may be easily adopted in order to build a more precise reference model, without impact over the controller design.

\footnotetext{
${ }^{1}$ Based on the tautology that for two real scalars $a$ and $b,(a-b)^{2} \geq 0$.
} 


\subsection{Damage compensator}

The damage compensator is designed to minimize the residue vector $\mathbf{e}_{\mathbf{x}}(t)$, based on modal adaptive strategy. Considering the model given in Eq. (5) and the control signal $\mathbf{u}(t)$ formed by the output signals of both controllers, it is possible to write the following system equation:

$$
\dot{\mathbf{x}}(t)=\mathbf{A x}(t)+\mathbf{B}_{1} \mathbf{w}(t)+\mathbf{B}_{\mathbf{2}} \mathbf{u}_{\mathbf{1}}(t)+\mathbf{B}_{\mathbf{2}} \mathbf{u}_{\mathbf{2}}(t)
$$

in which the pair $\left(\mathbf{A}, \mathbf{B}_{2}\right)$ is considered controllable.

Assuming an ideal fixed-gain control law for the damage compensator as:

$$
\mathbf{u}_{2}(t)=\left[\begin{array}{llll}
\mathbf{K}_{1}^{T} & \mathbf{K}_{2}^{T} & \cdots & \mathbf{K}_{\mathbf{m}}^{T}
\end{array}\right]\left[\begin{array}{c}
\mathbf{X}_{\mathbf{1}}(t) \\
\mathbf{X}_{\mathbf{2}}(t) \\
\vdots \\
\mathbf{X}_{\mathbf{m}}(t)
\end{array}\right]+\mathbf{K}_{\mathbf{u}_{\mathbf{1}}}^{T} \mathbf{u}_{\mathbf{1}}(t)=\mathbf{K}_{\mathbf{x}}^{T} \mathbf{x}(t)+\mathbf{K}_{\mathbf{u}_{\mathbf{1}}}^{T} \mathbf{u}_{\mathbf{1}}(t),
$$

in which $\mathbf{K}_{i}$ is the gain vector and $\mathbf{X}_{i}(t)$ is the state vector, both relative to mode $i$. Then, the closed-loop system with this controller is given by:

$$
\dot{\mathbf{x}}(t)=\left(\mathbf{A}+\mathbf{B}_{\mathbf{2}} \mathbf{K}_{\mathbf{x}}^{T}\right) \mathbf{x}(t)+\mathbf{B}_{\mathbf{1}} \mathbf{w}(t)+\left(\mathbf{B}_{\mathbf{2}}+\mathbf{B}_{\mathbf{2}} \mathbf{K}_{\mathbf{u}_{1}}^{T}\right) \mathbf{u}_{\mathbf{1}}(t)
$$

The damage compensator goal is to track asymptotically the reference model state to mitigate damage effects. The statespace model of the healthy structure is defined as:

$$
\dot{\mathbf{x}}_{\mathbf{r}}(t)=\mathbf{A}_{\mathbf{r}} \mathbf{x}_{\mathbf{r}}(t)+\mathbf{B}_{1 \mathbf{r}} \mathbf{W}(t)+\mathbf{B}_{2 \mathbf{r}} \mathbf{u}_{\mathbf{1}}(t),
$$

in which the matching conditions can be obtained by comparing Eqs. (19)-(20):

$$
\mathbf{A}_{\mathbf{r}}=\mathbf{A}+\mathbf{B}_{\mathbf{2}} \mathbf{K}_{\mathbf{x}}^{T}, \mathbf{B}_{1 \mathbf{r}}=\mathbf{B}_{1} \text {, and } \mathbf{B}_{\mathbf{2 r}}=\mathbf{B}_{\mathbf{2}}+\mathbf{B}_{\mathbf{2}} \mathbf{K}_{\mathbf{u}_{1}}^{T} \text {. }
$$

Based on Eq. (18) and the state-estimation results, the following real control law is proposed for the damage compensator:

$$
\mathbf{u}_{\mathbf{2}}(t)=\hat{\mathbf{K}}_{\mathbf{x}}^{T}(t) \hat{\mathbf{X}}(t)+\hat{\mathbf{K}}_{\mathbf{u}_{1}}^{T}(t) \mathbf{u}_{\mathbf{1}}(t)
$$

where $\hat{\mathbf{K}}_{\mathbf{x}}(t), \hat{\mathbf{K}}_{\mathbf{u}_{1}}(t)$, and $\hat{\mathbf{x}}(t)$ are respectively the online estimations of $\mathbf{K}_{\mathbf{x}}, \mathbf{K}_{\mathbf{u}_{1}}$, and $\mathbf{x}(t)$. Then, the closed loop with these new gains is given by:

$$
\dot{\mathbf{x}}(t)=\left(\mathbf{A}+\mathbf{B}_{\mathbf{2}} \hat{\mathbf{K}}_{\mathbf{x}}^{T}(t)\right) \hat{\mathbf{x}}(t)+\mathbf{B}_{1} \mathbf{w}(t)+\left(\mathbf{B}_{\mathbf{2}}+\mathbf{B}_{\mathbf{2}} \hat{\mathbf{K}}_{\mathbf{u}_{1}}^{T}(t)\right) \mathbf{u}_{\mathbf{1}}(t)
$$

\subsection{Reconfiguration mechanism}

The reconfiguration mechanism goal is to provide the online estimation of $\hat{\mathbf{K}}_{\mathbf{x}}(t)$ and $\hat{\mathbf{K}}_{\mathbf{u}_{1}}(t)$. For this purpose, the statetracking error dynamics is defined as $\dot{\mathbf{e}}_{\mathbf{x}}(t)=\dot{\mathbf{x}}(t)-\dot{\mathbf{x}}_{\mathbf{r}}(t)$. Substituting from Eqs. (20) and (22), and using the matching conditions, it leads to:

$$
\begin{aligned}
& \dot{\mathbf{e}}_{\mathbf{x}}(t)=\left(\mathbf{A}+\mathbf{B}_{\mathbf{2}} \hat{\mathbf{K}}_{\mathbf{x}}^{T}(t)\right) \hat{\mathbf{x}}(t)+\mathbf{B}_{\mathbf{1}} \mathbf{w}(t)+\left(\mathbf{B}_{\mathbf{2}}+\mathbf{B}_{\mathbf{2}} \hat{\mathbf{K}}_{\mathbf{u}_{1}}^{T}(t)\right) \mathbf{u}_{\mathbf{1}}(t)-\mathbf{A}_{\mathbf{r}} \mathbf{x}_{\mathbf{r}}(t)-\mathbf{B}_{\mathbf{1} \mathbf{r}} \mathbf{w}(t)-\mathbf{B}_{\mathbf{2}} \mathbf{\mathbf { u } _ { 1 }}(t)=\mathbf{A}_{\mathbf{r}} \mathbf{e}_{\mathbf{x}}(t)+\mathbf{B}_{\mathbf{2}} \Delta \mathbf{K}_{\mathbf{x}}^{T}(t) \hat{\mathbf{X}}(t) \\
& +\mathbf{B}_{\mathbf{2}} \Delta \mathbf{K}_{\mathbf{u}_{1}}^{T}(t) \mathbf{u}_{\mathbf{1}}(t),
\end{aligned}
$$

in which $\Delta \mathbf{K}_{\mathbf{x}}^{T}(t)=\left(\hat{\mathbf{K}}_{\mathbf{x}}^{T}(t)-\mathbf{K}_{\mathbf{x}}^{T}\right)$ and $\Delta \mathbf{K}_{\mathbf{u}_{1}}^{T}(t)=\left(\hat{\mathbf{K}}_{\mathbf{u}_{\mathbf{1}}}^{T}(t)-\mathbf{K}_{\mathbf{u}_{1}}^{T}\right)$ are the gain estimation errors.

The adaptive gains $\hat{\mathbf{K}}_{\mathbf{x}}(t)$ and $\hat{\mathbf{K}}_{\mathbf{u}_{1}}(t)$ must be adequately estimated in order to ensure the stability of the tracking error dynamics, aiming for the tracking error $\mathbf{e}_{\mathbf{x}}(t)$ to tend asymptotically to zero. To achieve these goals, the following theorem provides an online estimation of the gains.

Theorem 2. The state-tracking error dynamics given in Eq. (23) is stable for the following adaptive gain laws:

$$
\begin{aligned}
& \dot{\hat{\mathbf{K}}}_{\mathbf{x}}(t)=-\mathbf{T}_{\mathbf{x}} \hat{\mathbf{x}}(t) \mathbf{e}_{\mathbf{x}}^{T}(t) \mathbf{P B}_{\mathbf{2}}, \\
& \dot{\hat{\mathbf{K}}}_{\mathbf{u}_{\mathbf{1}}}(t)=-\mathbf{T}_{\mathbf{u}_{1}} \mathbf{u}_{\mathbf{1}}(t) \mathbf{e}_{\mathbf{x}}^{T}(t) \mathbf{P B} \mathbf{B}_{\mathbf{2}},
\end{aligned}
$$

in which for $\mathbf{R}=\mathbf{R}^{T}>0, \mathbf{P}=\mathbf{P}^{T}>0$ satisfies the following algebraic Lyapunov equation: 


$$
\mathbf{P A}+\mathbf{A}_{\mathbf{r}}^{T} \mathbf{P}=-\mathbf{R}
$$

Moreover, $\mathbf{T}_{\mathbf{x}}>0$ and $\mathbf{T}_{\mathbf{u}_{\mathbf{1}}}>0$ are diagonal matrices that determine adaptation rates. Matrix $\mathbf{T}_{\mathbf{x}}$ is a function of the modal adaptation-rate submatrices:

$$
\mathbf{T}_{\mathbf{x}}=\left[\begin{array}{cccc}
\mathbf{T}_{1} & \mathbf{0} & \cdots & \mathbf{0} \\
\mathbf{0} & \mathbf{T}_{2} & \cdots & \mathbf{0} \\
\vdots & \vdots & \ddots & \vdots \\
\mathbf{0} & \mathbf{0} & \mathbf{0} & \mathbf{T}_{\mathbf{m}}
\end{array}\right]
$$

where the $2 \times 2$ matrix $\mathbf{T}_{i}$ determines the adaptation rate of mode $i$.

Proof. The following quadratic Lyapunov candidate function is chosen to analyze the stability of the state-tracking error dynamics:

$$
V\left(\mathbf{e}_{\mathbf{x}}(t), \Delta \mathbf{K}_{\mathbf{x}}(t), \Delta \mathbf{K}_{\mathbf{u}_{1}}(t)\right)=\mathbf{e}_{\mathbf{x}}^{T}(t) \mathbf{P} \mathbf{e}_{\mathbf{x}}(t)+\operatorname{tr}\left(\left[\Delta \mathbf{K}_{\mathbf{x}}^{T}(t) \mathbf{T}_{\mathbf{x}}^{-1} \Delta \mathbf{K}_{\mathbf{x}}(t)\right]+\operatorname{tr}\left(\left[\Delta \mathbf{K}_{\mathbf{u}_{1}}^{T}(t) \mathbf{T}_{\mathbf{u}_{1}}^{-1} \Delta \mathbf{K}_{\mathbf{u}_{1}}(t)\right]\right),\right.
$$

in which $t r$ represents the trace of a matrix.

The derivative of $V\left(\mathbf{e}_{\mathbf{x}}(t), \Delta \mathbf{K}_{\mathbf{x}}(t), \Delta \mathbf{K}_{\mathbf{u}_{1}}(t)\right)$, evaluated using Eq. (23), is given by:

$$
\begin{aligned}
& \dot{V}\left(\mathbf{e}_{\mathbf{x}}(t), \Delta \mathbf{K}_{\mathbf{x}}(t), \Delta \mathbf{K}_{\mathbf{u}_{\mathbf{1}}}(t)\right)=\mathbf{e}_{\mathbf{x}}^{T}(t)\left(\mathbf{A}_{\mathbf{r}}^{T} \mathbf{P}+\mathbf{P A}_{\mathbf{r}}\right) \mathbf{e}_{\mathbf{x}}(t)+2 \mathbf{e}_{\mathbf{x}}^{T}(t) \mathbf{P} \mathbf{B}_{\mathbf{2}} \Delta \mathbf{K}_{\mathbf{x}}^{T}(t) \hat{\mathbf{X}}(t)+2 \operatorname{tr}\left(\left[\Delta \mathbf{K}_{\mathbf{x}}^{T}(t) \mathbf{T}_{\mathbf{x}}^{-1} \dot{\hat{\mathbf{K}}} \mathbf{x}_{\mathbf{x}}(t)\right]\right)+2 \mathbf{e}_{\mathbf{x}}^{T}(t) \mathbf{P} \mathbf{B}_{\mathbf{2}} \Delta \mathbf{K}_{\mathbf{u}_{\mathbf{1}}}^{T}(t) \mathbf{u}_{\mathbf{1}}(t) \\
& +2 \operatorname{tr}\left(\left[\Delta \mathbf{K}_{\mathbf{u}_{\mathbf{1}}}^{T}(t) \mathbf{T}_{\mathbf{u}_{\mathbf{1}}}^{-1} \dot{\hat{\mathbf{K}}}_{\mathbf{u}_{\mathbf{1}}}(t)\right]\right)=-\mathbf{e}_{\mathbf{x}}^{T}(t) \mathbf{R e}_{\mathbf{x}}(t)+2 \operatorname{tr}\left(\Delta \mathbf{K}_{\mathbf{x}}^{T}(t)\left[\hat{\mathbf{x}}(t) \mathbf{e}_{\mathbf{x}}^{T}(t) \mathbf{P B}_{\mathbf{2}}+\mathbf{T}_{\mathbf{x}}^{-1} \dot{\hat{\mathbf{K}}} \mathbf{x}_{\mathbf{x}}(t)\right]\right)+2 \operatorname{tr}\left(\Delta \mathbf{K}_{\mathbf{u}_{1}}^{T}(t)\right. \\
& \left.\left[\mathbf{u}_{1}(t) \mathbf{e}_{\mathbf{x}}^{T}(t) \mathbf{P B}_{2}+\mathbf{T}_{\mathbf{u}_{1}}^{-1} \dot{\hat{\mathbf{K}}}_{\mathbf{u}_{1}}(t)\right]\right) .
\end{aligned}
$$

If the adaptive laws are selected as:

$$
\dot{\hat{\mathbf{K}}}_{\mathbf{x}}(t)=-\mathbf{T}_{\mathbf{x}} \hat{\mathbf{x}}(t) \mathbf{e}_{\mathbf{x}}^{T}(t) \mathbf{P} \mathbf{B}_{\mathbf{2}} \text { and } \dot{\hat{\mathbf{K}}}_{\mathbf{u}_{\mathbf{1}}}(t)=-\mathbf{T}_{\mathbf{u}_{\mathbf{1}}} \mathbf{u}_{\mathbf{1}}(t) \mathbf{e}_{\mathbf{x}}^{T}(t) \mathbf{P} \mathbf{B}_{\mathbf{2}},
$$

then $\dot{V}\left(\mathbf{e}_{\mathbf{x}}(t), \Delta \mathbf{K}_{\mathbf{x}}(t), \Delta \mathbf{K}_{\mathbf{u}_{1}}(t)\right)=-\mathbf{e}_{\mathbf{x}}^{T}(t) \mathbf{R e}_{\mathbf{x}}(t) \leq 0$. Therefore, the state-tracking error tends asymptotically to zero, i.e., $\lim _{t \rightarrow \infty}\left\|\hat{\mathbf{x}}(t)-\mathbf{x}_{\mathbf{r}}(t)\right\|=0$. This proves the theorem.

\subsection{Modal double-loop controller design procedure}

The procedure to design the modal double-loop controller can be divided into two parts: the nominal controller design given in Algorithm 1 and the damage compensator design given in Algorithm 2.

\section{Algorithm 1. Nominal controller design.}

1: Determine the modal state-space model of the healthy plant using Eqs. (4) and (5);

2: Design a regular $H_{\infty}$ controller using the traditional approach. Then, adjust the weighing filters, based on Eq. (9);

3: Choose the modes to act upon, based on the modal performance of the standard $H_{\infty}$ controller; build the modal weighing matrices; and build the respective modal performance indicator vector (Eq. (8));

4: Solve the modal $H_{\infty}$ problem.

\section{Algorithm 2. Damage compensator design.}

1: Design the modal observer by solving the respective LMI (12);

2: Design the bandpass filters;

3: Build the reference model, using the closed loop of the healthy structure model given in Eq. (5) under the nominal controller represented in Eq. (6);

4: Determine matrix $\mathbf{R}>0$. After that, compute the matrix $\mathbf{P}$ by solving the algebraic Lyapunov equation given in Eq. (26);

5: Define the matrix $\mathbf{T}_{\mathbf{u}_{\mathbf{1}}}$ and the modal matrices $\mathbf{T}_{\mathbf{i}}$ to build $\mathbf{T}_{\mathbf{x}}$, following Eq. (27);

6: Build the adaptive gain laws (Eqs. (24) and (25));

7: Build the control law (Eq. (21)).

\section{Simulated results}

This section shows results that permit to analyze the proposed methodology effectiveness. In the sequence, a flexible structure presenting two severity levels of damage is examined, based on FE modeling. After that, a modal $H_{\infty}$ controller (MC) and a modal adaptive controller are designed to compose the modal double-loop framework (MDLF). A regular $H_{\infty}$ controller $(\mathrm{RC})$ is also designed to be included in the evaluation of the proposed framework. The performances of the regular and the nominal controllers in the single loop are compared and analyzed with the modal double-loop controller. 


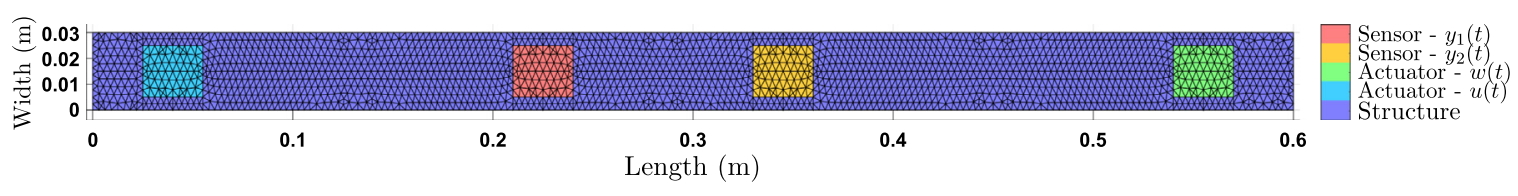

(a) Flexible structure.

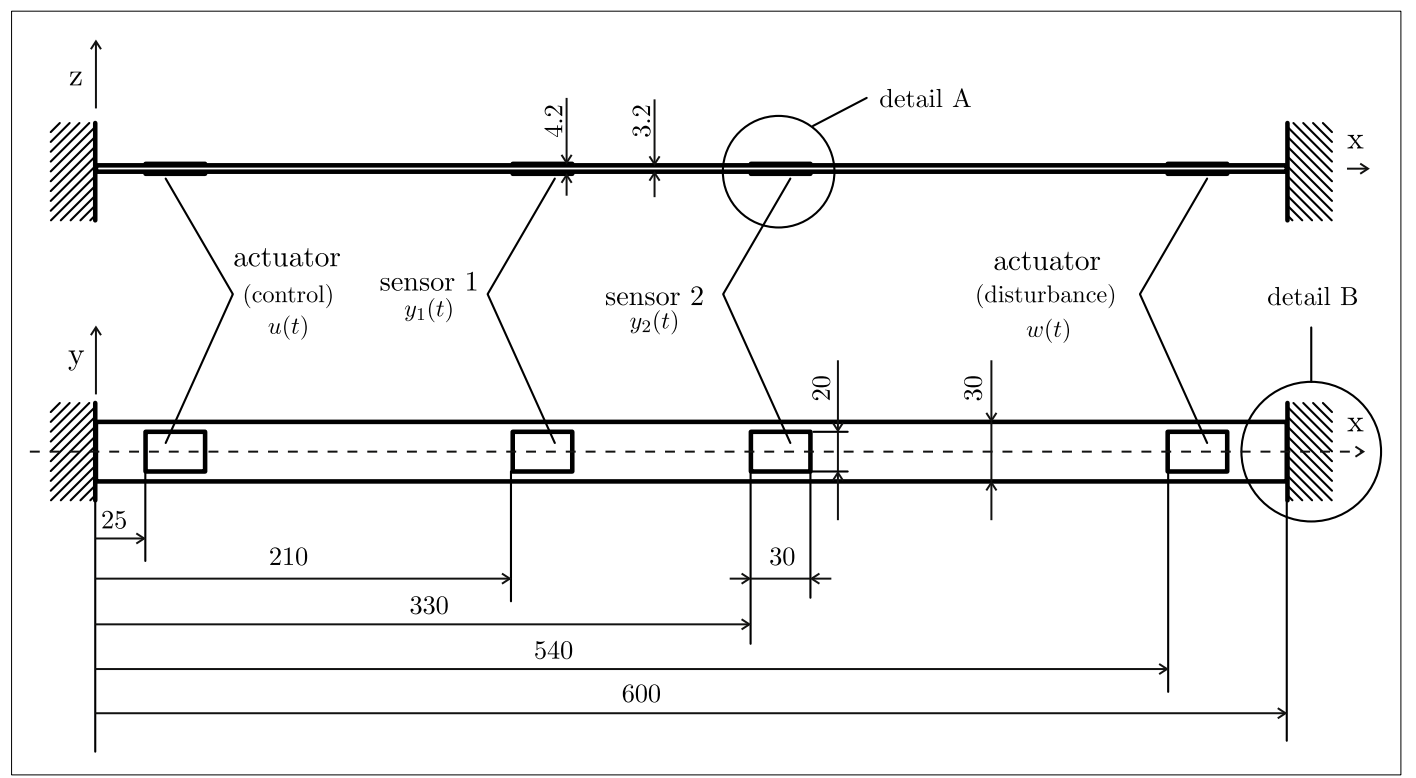

(b) Schematic diagram in $\mathrm{mm}$.

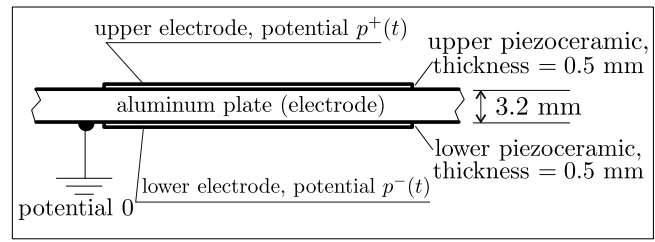

(c) Detail A.

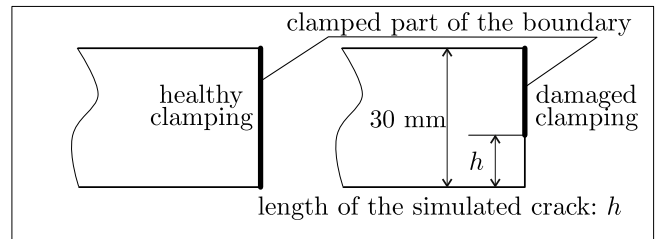

(d) Detail B.

Fig. 6. Adopted aluminum structure with active elements.
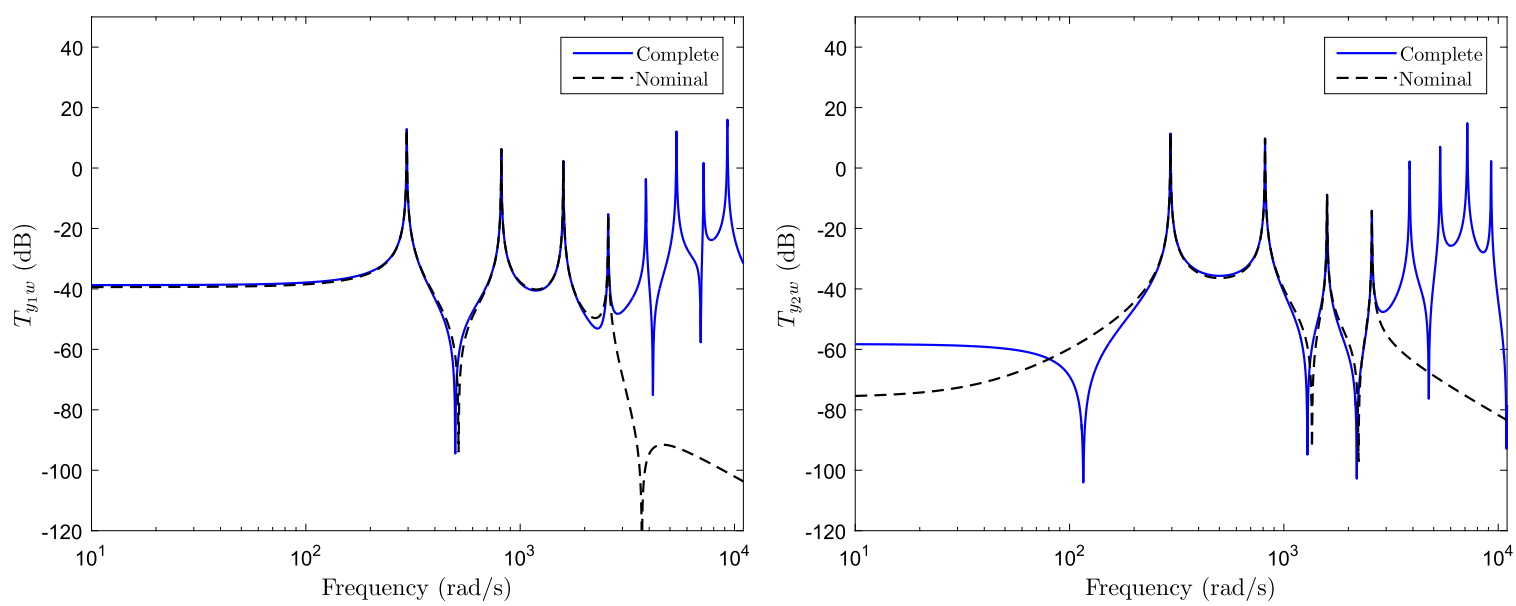

Fig. 7. Transfer function comparison between complete and nominal models. 
Table 1

First natural frequencies of the healthy structure.

\begin{tabular}{lllll}
\hline Mode & Frequency (rad/s) & Deformation & Mode & Fequency (rad/s) \\
\hline 1 & 295.69 & Bending & 6 & 3861.10 \\
2 & 816.72 & Bending & 7 & 5360.21 \\
3 & 1590.10 & Bending & 8 & 7173.37 \\
4 & 2574.19 & Bending & 9 & 7630.50 \\
5 & 3683.19 & Torsion & 10 & 9267.03 \\
\hline
\end{tabular}

A clamped-clamped aluminum beam of Fig. 6a with a rectangular cross section of $30 \mathrm{~mm} \times 3.2 \mathrm{~mm}$ is adopted to examine the modal double-loop methodology, according to dimensions and elements in Fig. 6b. Four pairs of piezoelectric ceramic elements (PZTs) are fixed symmetrically to each side of the beam and used as transducers, following the guidelines presented in Fig. 6c. The simulated beam is created using a dedicated FE software developed at PIMM Laboratory to test control strategies on plate structures. The FE model mesh contains 3939 discrete Kirchhoff triangular plate elements [54], considering 472 ones to model the four areas where the piezoelectric transducers are placed. The layers are used to model the structure with the respective transducers, one layer representing the structure and the other is used to describe each piezoelectric ceramic, see [55] for more details. Appendix A contains the material properties of the active structure.

The nominal plant model used to design the controllers and the observer is reduced to four modes. Another model with ten modes, referred here as the complete model, is adopted to analyze the controller performances, in order to verify that the controllers are effective in avoiding the spillover. The comparison of the open-loop transfer functions for both models is presented in Fig. 7. The respective vibration modes and natural frequencies are presented in Table 1.

\subsection{Damage simulation}

A crack of length $h$ localized at the beam extremity is used to simulate two levels of damage, considering two different lengths following Fig. 6d:

- No damage: $h=0$;

- Damage 1: $h=15 \mathrm{~mm}$;

- Damage 2: $h=20 \mathrm{~mm}$.

Damage effects can be noted in Fig. 8, where the frequency response comparison between the healthy and damaged structures is shown, considering damage 2 . It is possible to verify that the damage produces natural frequency shifts and amplitude variation in the four modes. For the first three modes, the damage reduces the peak amplitudes, with mode 3 presenting the largest attenuation. However, damage effect on mode 4 is peak amplification, even with this mode presenting the lowest energy vibration in the healthy structure.

\subsection{Nominal controller design}

The nominal controller is designed based on the nominal model of the healthy structure, where the performance matrices are defined as $\mathbf{C}_{\mathbf{1}}=\mathbf{C}_{\mathbf{2}}$ and $\mathbf{D}_{\mathbf{1 1}}=\mathbf{D}_{\mathbf{1 2}}=\left[\begin{array}{ll}0 & 0\end{array}\right]^{T}$. A chirp signal, with bandwidth from $0 \mathrm{~Hz}$ to $500 \mathrm{~Hz}$, duration of $20 \mathrm{~s}$,
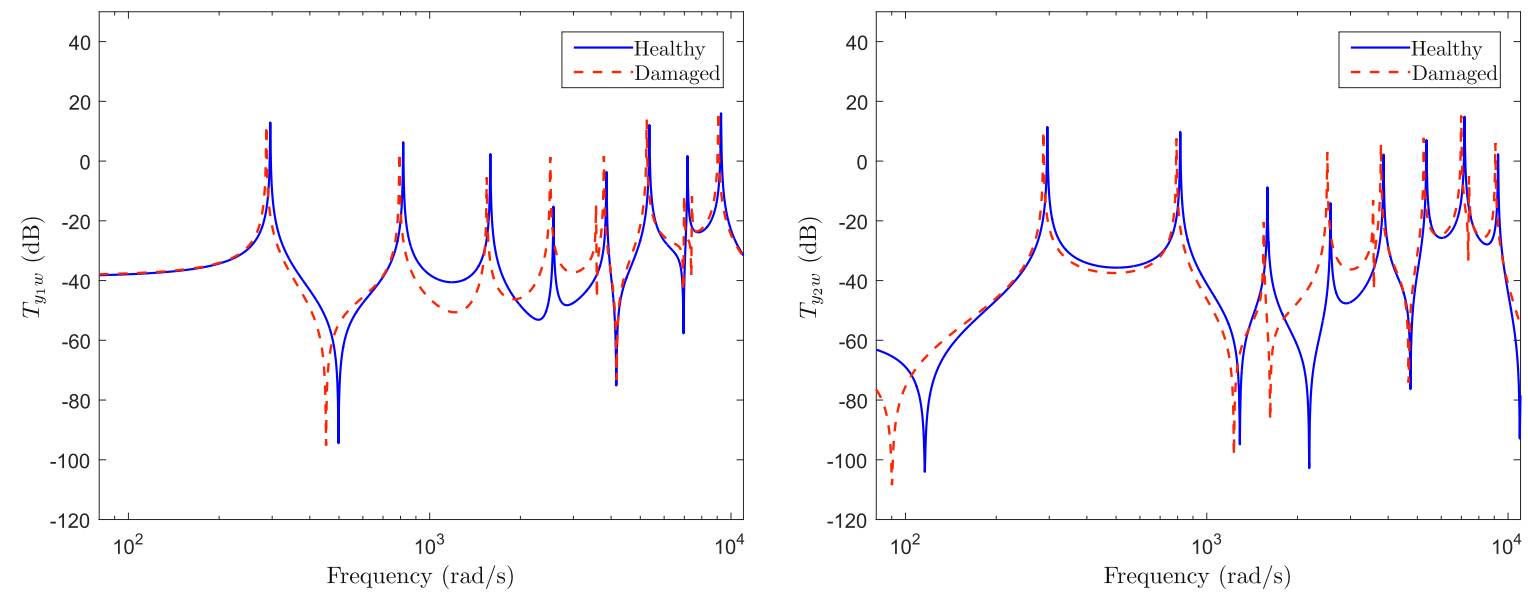

Fig. 8. Frequency response comparison between healthy and damaged structures (damage 2). 
Table 2

Parameters of the weighing filters.

\begin{tabular}{llrr}
\hline Weighing filters & $\omega_{\mathrm{c}}(\mathrm{rad} / \mathrm{s})$ & $k$ & \multicolumn{1}{c}{ M } \\
\hline$F_{u}$ & 2320 & 2 & 65 \\
$F_{\mathbf{z}}$ & 2320 & 1 & 0.07 \\
\hline
\end{tabular}
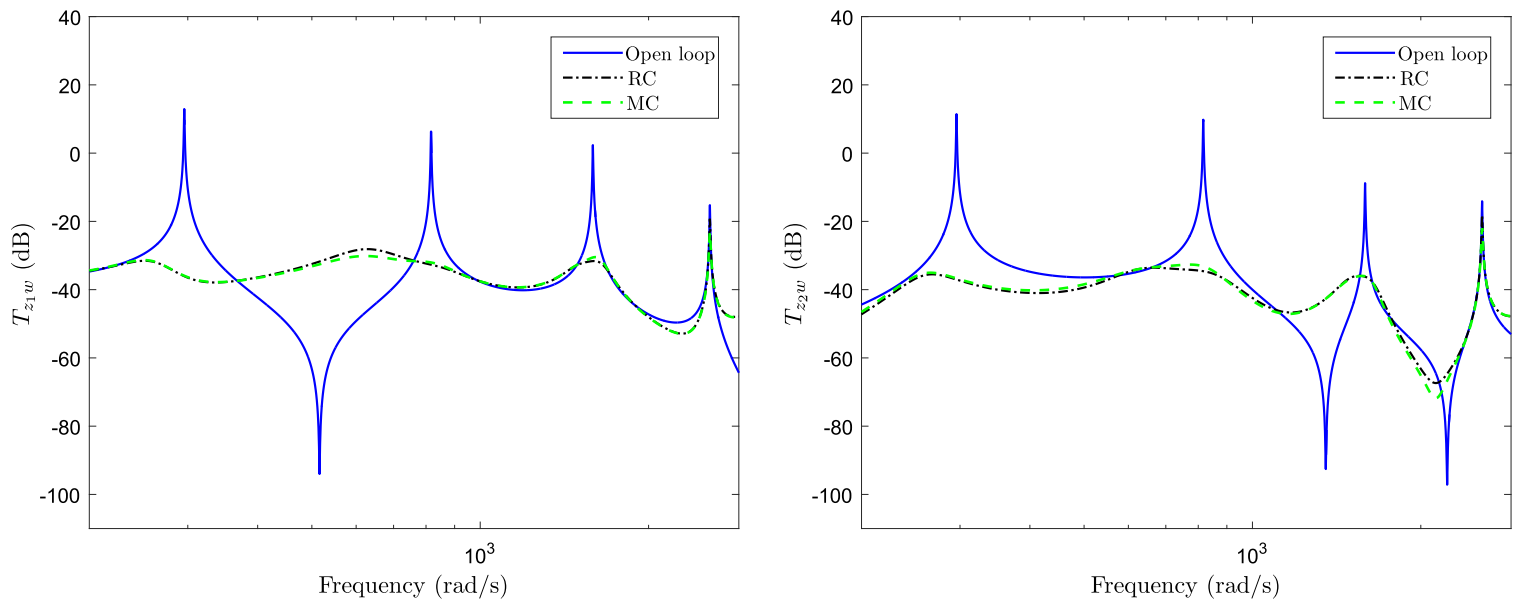

Fig. 9. Frequency response comparison between the uncontrolled and the controlled healthy structure.
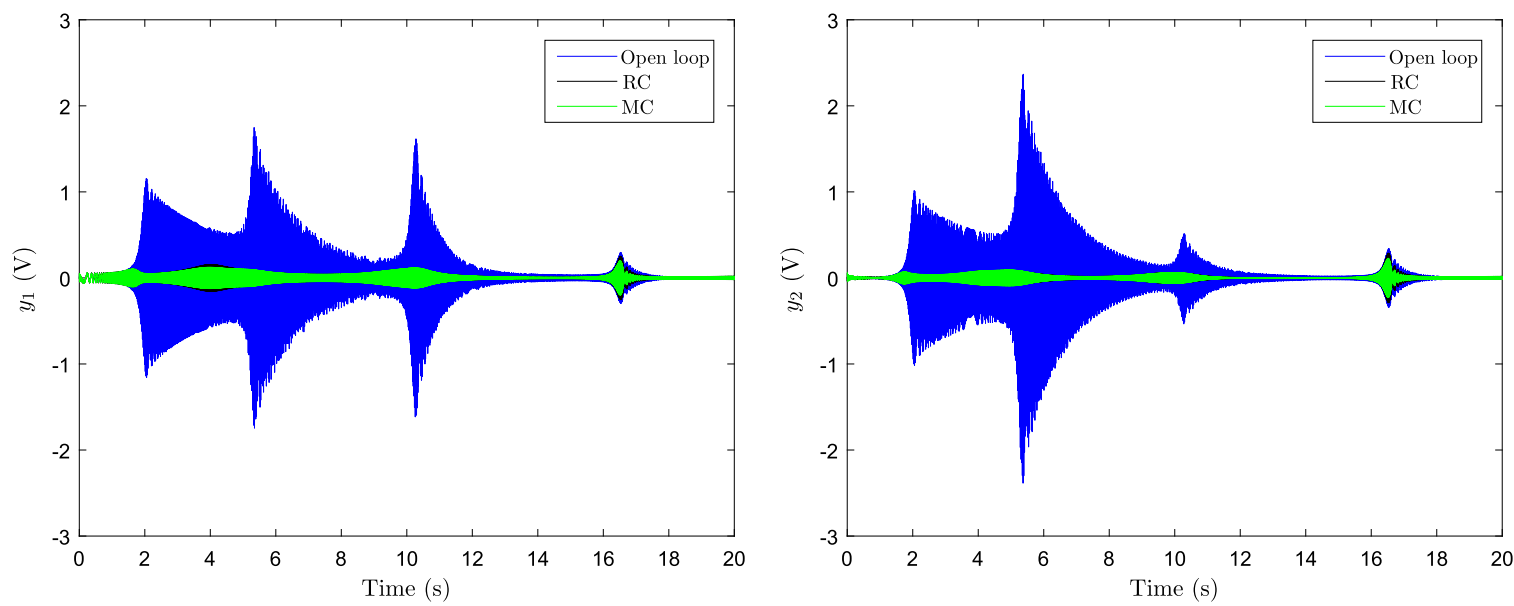

Fig. 10. Performance comparison between the uncontrolled and the controlled healthy structure.

and amplitude of $4 \mathrm{~V}$, is considered as a disturbance in order to examine the controller performance. To balance the control signals and the relation between disturbance and performance signals, the weighing filters are designed with the parameters presented in Table 2. Moreover, one can notice that the filter $F_{u}$ also limits the generated control signal.

The RC is initially designed to reduce the vibration of the healthy structure and uses the same filters of the nominal controller in order to have an acceptable comparison base. This controller is used to provide reference performances to be compared with the other control techniques. Frequency and time responses for the open-loop and closed-loop healthy structure are shown in Figs. 9 and 10, which also show results for the MC presented next. It is possible to see that the RC reduces the peak vibration for all modes, with modes 1 and 2 presenting the largest reduction.

The nominal controller is designed based on the modal approach with the same controller structure of the RC, adding the respective performance outputs. These matrices are built considering the low peak vibration attenuation of mode 4 , obtained by the RC. Thus, the following weighing matrices are adopted:

$$
\mathbf{Q}_{1}^{\frac{1}{2}}=\left[\begin{array}{cc}
0.7 & 0 \\
0 & 0.7
\end{array}\right], \quad \mathbf{Q}_{2}^{\frac{1}{2}}=\left[\begin{array}{cc}
0.7 & 0 \\
0 & 0.7
\end{array}\right], \quad \mathbf{Q}_{3}^{\frac{1}{2}}=\left[\begin{array}{cc}
0.9 & 0 \\
0 & 0.9
\end{array}\right], \quad \text { and } \quad \mathbf{Q}_{4}^{\frac{1}{2}}=\left[\begin{array}{cc}
1.6 & 0 \\
0 & 1.6
\end{array}\right]
$$

The performance of both single-loop controllers for the healthy structure is compared in Figs. 9 and 10. Considering 


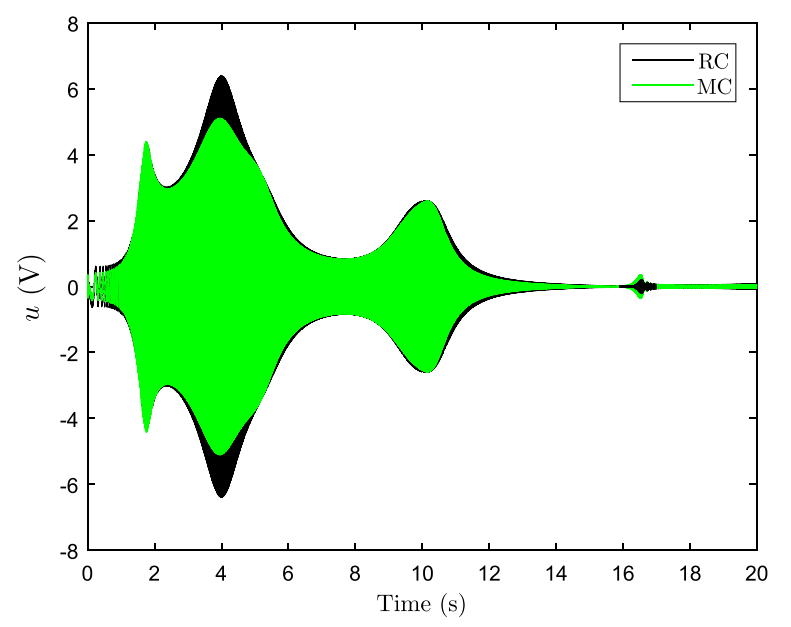

Fig. 11. Control signal comparison between the MC and the RC in the healthy structure.

modes 1 and 2, the RC performance is slightly higher than the MC performance due to the low weight of the MC. For mode 3 , both controllers have similar performances due to the small change of the respective modal weighing matrix. However, the MC performance is better than the RC one for mode 4, due to the respective modal weighing increase. Fig. 11 permits to analyze the modal control energy distribution of both controllers, where the color superposition changes in order to clarify the relative amplitudes. Comparing with the RC, the MC control signal has its amplitude reduced in mode 2, does not change in modes 1 and 3, and is amplified in mode 4. It is possible to affirm that both controllers have a satisfactory performance considering the healthy structure vibration attenuation.

\subsection{Damage compensator design}

Following the Algorithm 2 to design the damage compensator, the first step is to design the modal observer, solving the LMI (12), where it is adopted $\nu=5$ and $\alpha=5$. A Butterworth approach is adopted for the bandpass filters, according to the parameters presented in Table 3.

The matrix $R$ and the modal adaptation-rate matrices are chosen with the highest gains over the modes 3 and 4 , due to the low vibration attenuations achieved with the MC. Thus, the following matrices are adopted:

$$
\begin{aligned}
& \mathbf{R}=\operatorname{diag}(0.01 ; 0.01 ; 0.01 ; 0.01 ; 0.1 ; 0.1 ; 1 ; 1), \mathbf{T}_{\mathbf{1}}=\mathbf{T}_{\mathbf{2}}=\left[\begin{array}{cc}
50 & 0 \\
0 & 50
\end{array}\right], \mathbf{T}_{\mathbf{3}}=\left[\begin{array}{cc}
500 & 0 \\
0 & 500
\end{array}\right], \mathbf{T}_{\mathbf{4}}=\left[\begin{array}{cc}
2000 & 0 \\
0 & 2000
\end{array}\right], \\
& \quad \text { and } \mathbf{T}_{u_{1}}=0.001
\end{aligned}
$$

The adaptive controller struggles to track the reference model state vector by estimating the gains $\mathbf{K}_{\mathbf{x}}$ and $\mathbf{K}_{\mathbf{u}_{1}}$. The control effort may generate high amplitude signals, leading even to actuator saturation, however, producing a small reduction of the state-tracking error. A threshold is adopted to interrupt the iteration process, setting the gains to the last value. This procedure is described by the following rule:

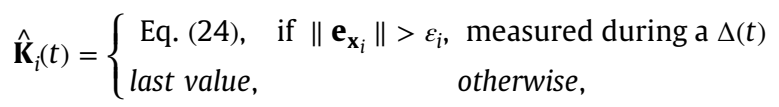

where $\Delta(t)$ is a specific time period and $\hat{\mathbf{K}}_{i}, \mathbf{e}_{\mathbf{x}_{i}}$, and $\varepsilon_{\mathrm{i}}$ represent respectively the estimation gains, the state-vector difference, and the threshold relative to mode $i$.

\subsection{Controller responses under damage}

The same chirp signal adopted before is used to simulate the disturbance for three cycles of twelve repetitions of the

Table 3

Parameters of the bandpass filters.

\begin{tabular}{lllll}
\hline Cutoff frequency (rad/s) & Mode 1 & Mode 2 & Mode 3 & Mode 4 \\
\hline$\omega_{c_{1}}$ & 0 & 400 & 1200 & 1900 \\
$\omega_{c_{2}}$ & 400 & 1200 & 1900 & 2700 \\
\hline
\end{tabular}



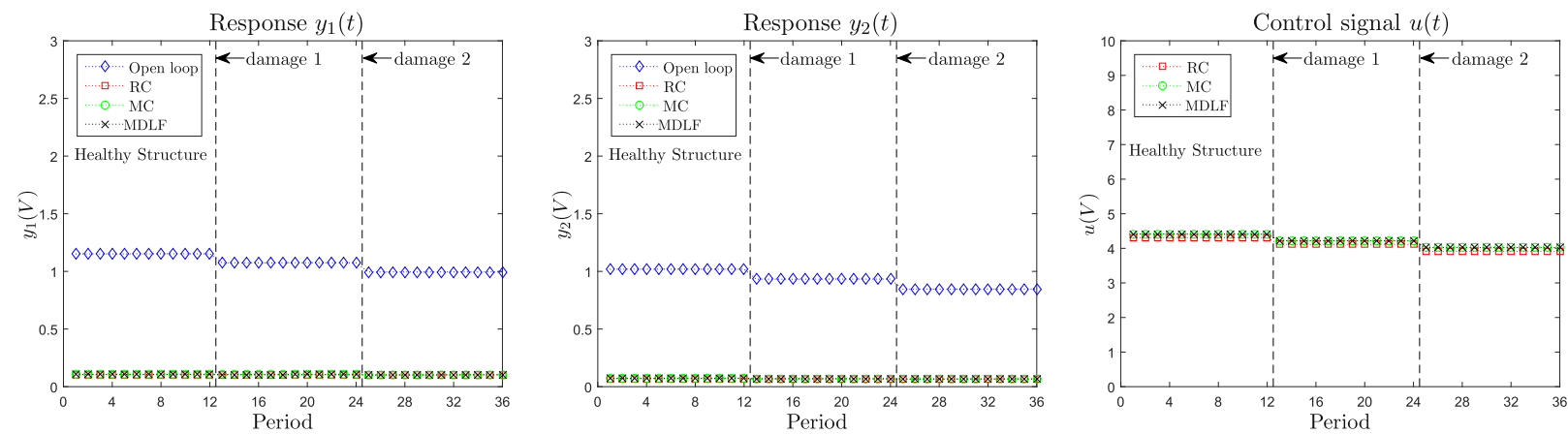

(a) Mode 1.
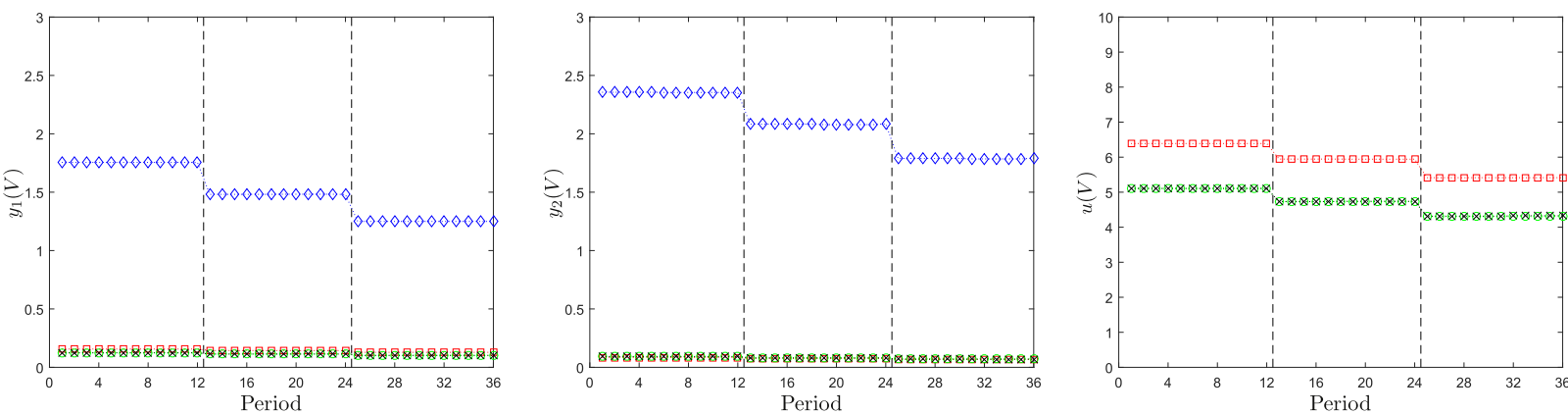

(b) Mode 2 .
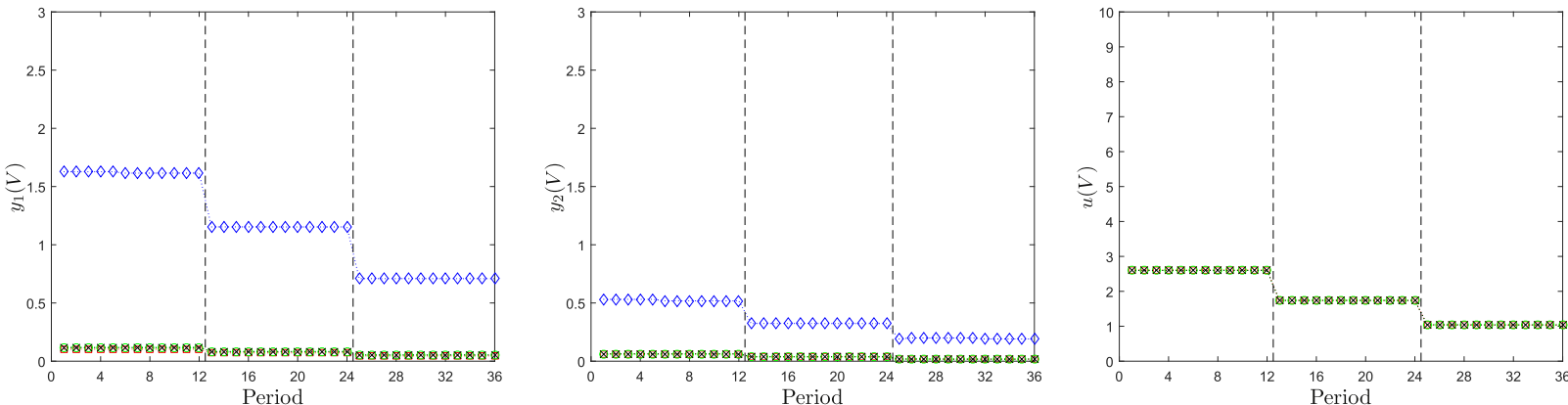

(c) Mode 3 .
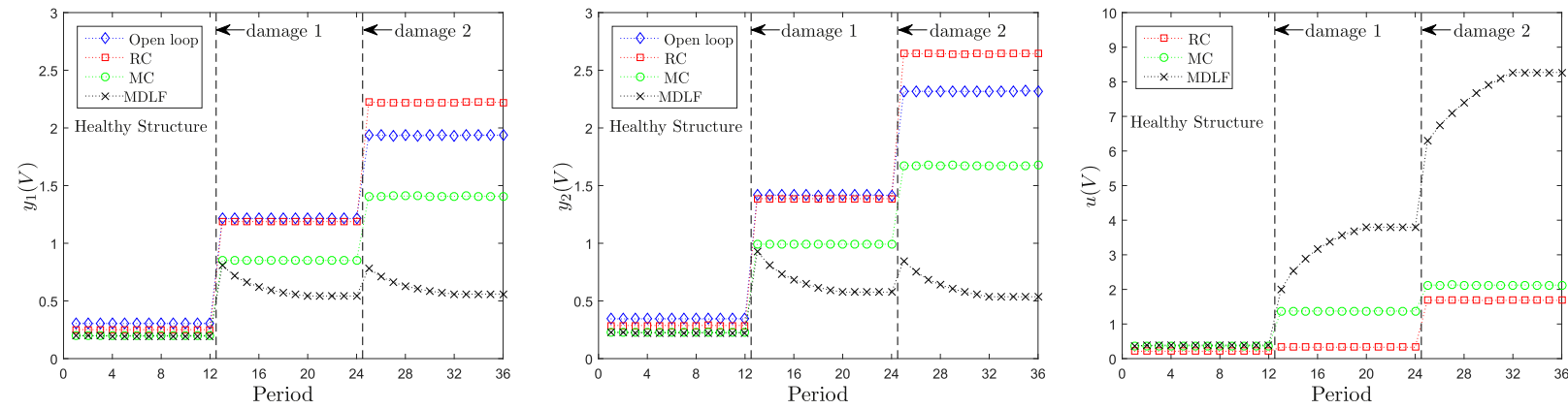

(d) Mode 4.

Fig. 12. Modal peak performance comparison of the controlled structure submitted to damage.

signal. Each cycle corresponds to one condition of the structure: healthy plant, plant with damage 1, and plant with damage 2 , as described before. Three controller methodologies are examined in these different situations: single-loop RC, singleloop MC, and the MDLF formed by the composition of the MC and the adaptive controller.

Fig. 12 shows the behavior of each mode in twelve separate panels. Modal peak amplitudes from the open-loop response 


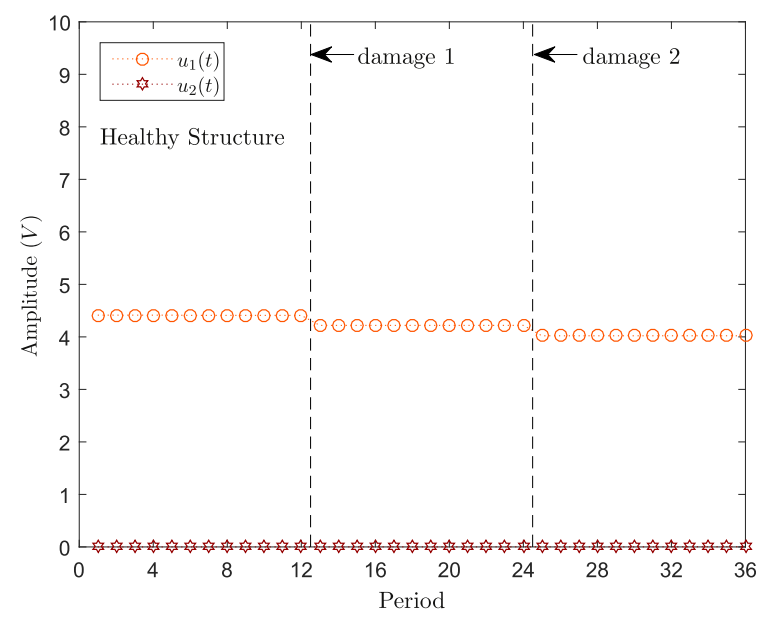

(a) Mode 1 .

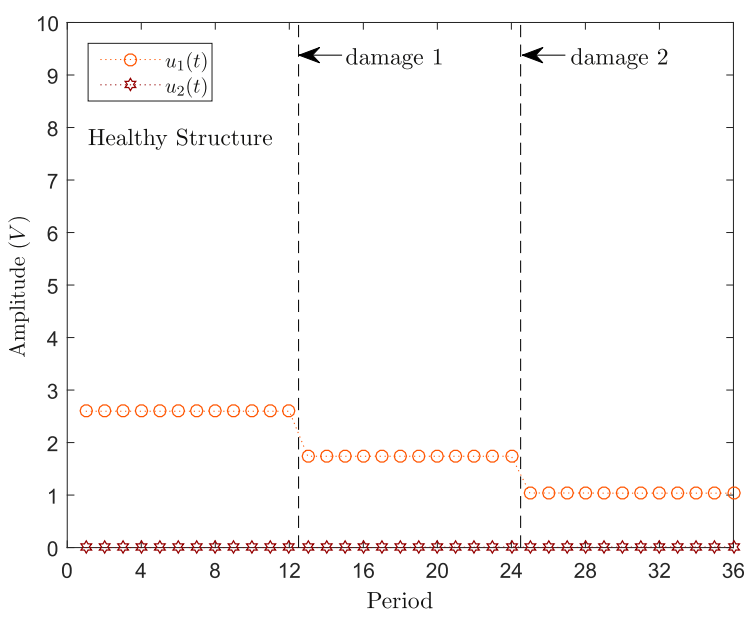

(c) Mode 3 .

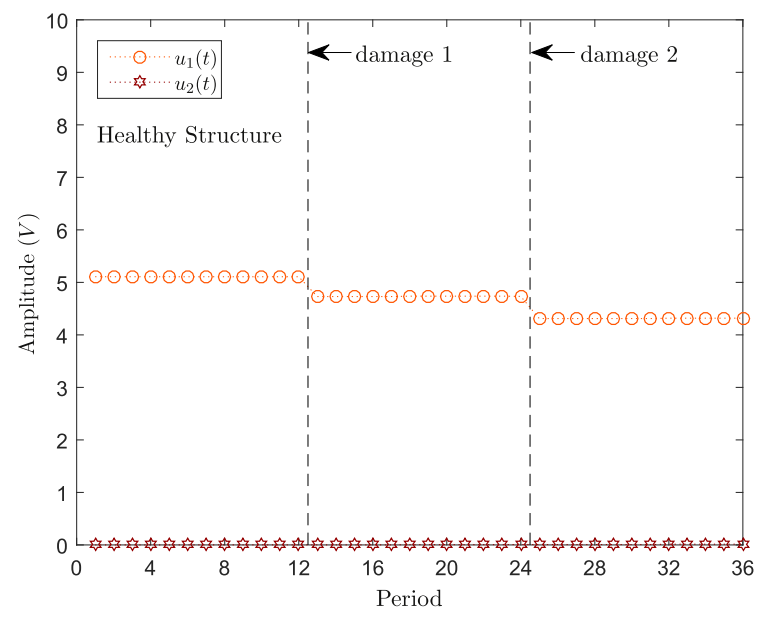

(b) Mode 2 .

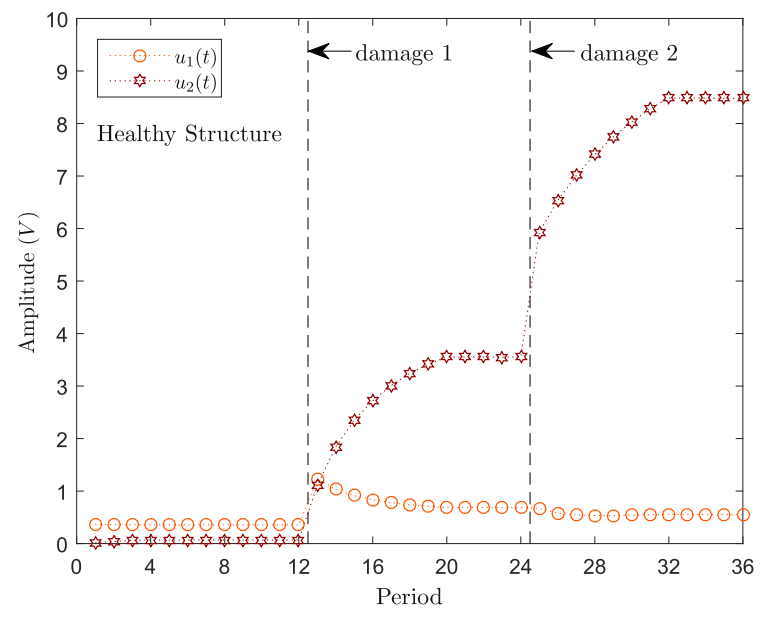

(d) Mode 4.

Fig. 13. Control signal components of the MDLF.

and also the closed-loop response for the output signals $y_{1}(t)$ and $y_{2}(t)$, for each one of the three adopted controllers, and the three respective control signal $u(t)$ may be seen in the panels.

It is possible to see that damage occurrence reduces the amplitude of modes 1-3 of the uncontrolled structure as previously discussed. However, damage does not change the controller performances in modes $1-3$, for both levels of damage severity. Regarding the control signals, the peak amplitudes are similar to all controllers for modes 1 and 3. For mode 2, it may be seen that the control signal amplitude for the RC controller is higher than those for both modal controllers. This shows modal controllers efficiency over the RC. Regarding mode 4, the open-loop vibration amplitude increases with damage severity and the controllers have distinct performances. The respective modal vibration increases significantly under the RC, getting even higher than the open-loop respective peak. The MC presents a better performance than the RC, however, the modal vibrations also increase with damage severity. For damage 1, the MDLF initially has a response similar to the MC but the adaptive adjustment of its parameters gradually reduces the peak amplitude. For damage 2 , the response is much better since the beginning of the damage occurrence. The control signal amplitudes increase for all controllers to face damage, however, the MDLF amplitude is the highest, which leads to the efficient attenuation of the mode.

Fig. 13 presents the modal peak amplitudes of the two control signals $u_{1}(t)$ and $u_{2}(t)$, aiming to analyze the contribution of the nominal controller and the damage compensator to the general performance of the MDLF. It may be seen that $u_{1}(t)$, generated by the nominal controller, is the main responsible for attenuating the vibrations of the first three modes, presenting a similar performance for the three plant conditions while maintaining $u_{2}(t)$ very small. Regarding mode 4 , this behavior is inverted, and the main contribution for the control signal is now from $u_{2}(t)$. It may be seen that the control signal of the nominal controller increases a little with damage, but the high level of $u_{2}(t)$ dominates these signals. Considering the overall performance for all modes, it is possible to affirm that both controllers cooperate to achieve a satisfactory performance. 


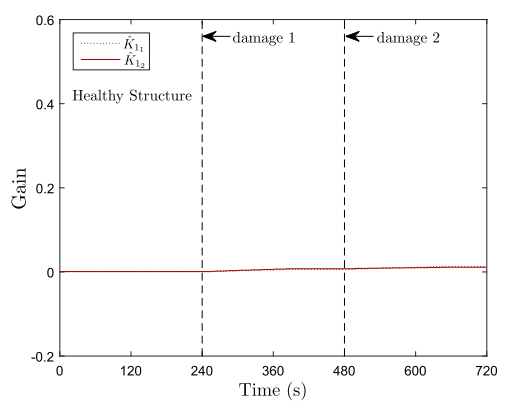

(a) Mode 1.

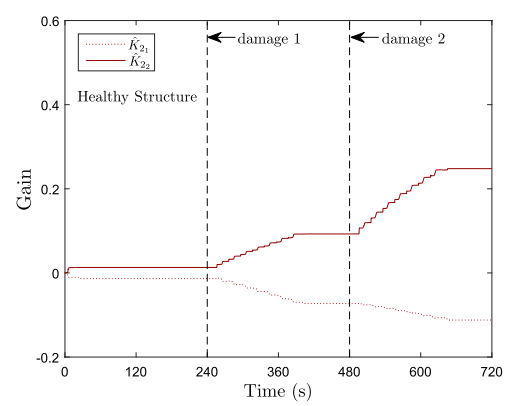

(b) Mode 2 .

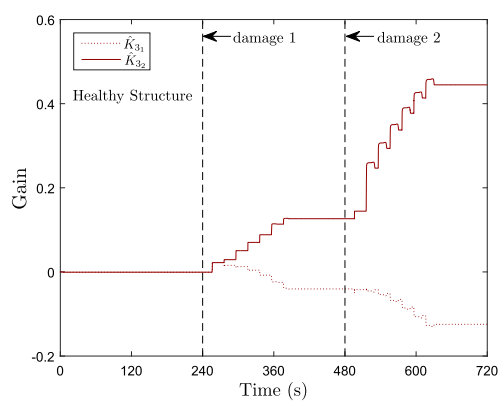

(c) Mode 3.

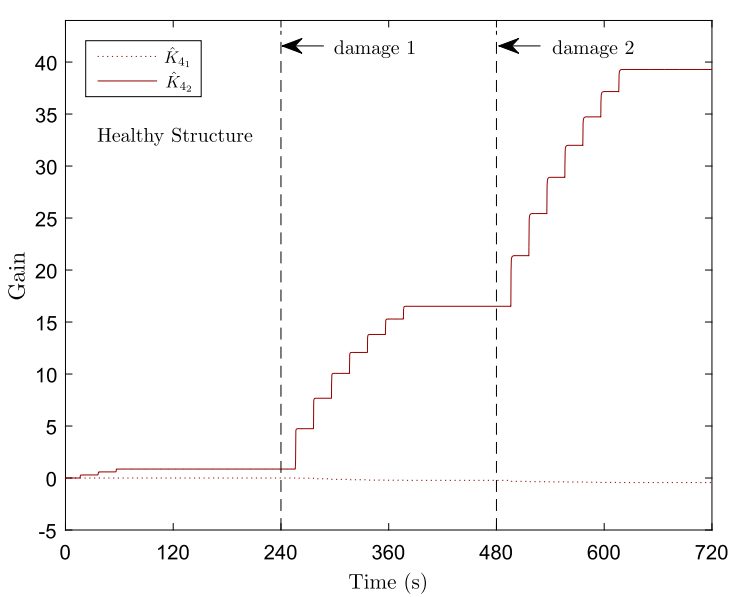

(d) Mode 4 .

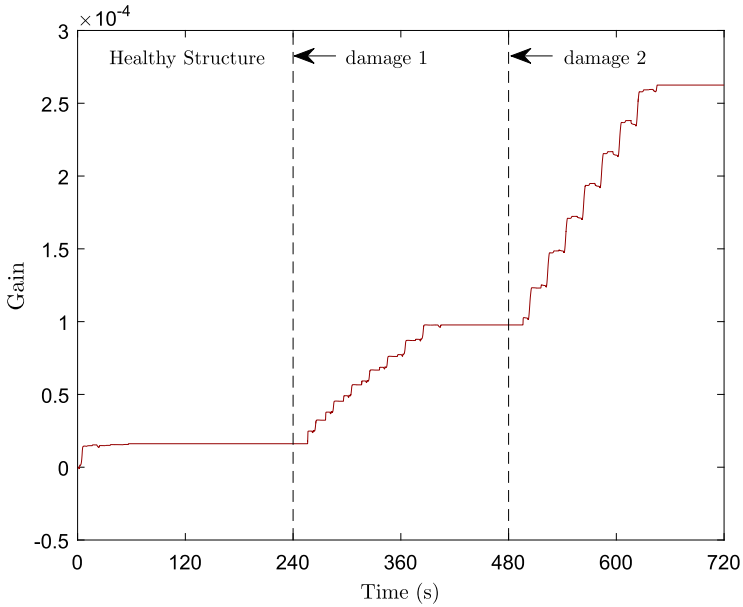

(e) $\hat{K}_{u_{1}}$.

Fig. 14. Adaptive gains of the damage compensator

Considering that the damage compensator has two parameters, in which the first is the modal gain vector that multiplies the state vector and the second is a scalar that weighs the nominal control signal, the adaptive evolution of these parameters is analyzed based on the signals represented in Fig. 14. It may be seen that, despite the fact that the gains of mode 3 are larger than mode 2 gains, which are also larger than mode 1 gains, in reaction to damage severity, all these values are very small compared to mode 4 gains. One can notice the difference in the vertical scales of these figures. To conclude, Fig. 14e shows that the contribution of the gain $\hat{\mathbf{K}}_{u_{1}}$ to the composition of $u_{2}(t)$ is very small.

\section{Conclusion}

A new control methodology for damage-tolerant active control is presented in this paper, based on a modal double-loop control framework. In the outer loop, a modal $H_{\infty}$ controller is designed to comply with performance and robustness requirements for the healthy structure. The second modal controller, reconfigured online, has the objective of compensating damage effects through online controller parameter adaptation, based on the output of a modal observer. The damage compensator objective is to reduce the state-tracking error between the closed-loop performances of the healthy and the damaged structure, aiming to mitigate the increasing vibration caused by damage.

The proposed methodology is examined using the FE modeling of a clamped-clamped structure, where the controlled responses for the healthy plant and for two different damage severity levels were simulated. A regular $H_{\infty}$ controller, a single-loop modal $H_{\infty}$ controller, and the modal double-loop framework were adopted and their performances facing damage were compared. Results show that the modal double-loop framework is the most effective in mitigating the damage effects on structural vibrations, based on the effective response of the damage compensator. Moreover, the modal doubleloop framework had also an adequate performance for the healthy structure, due to the modal $H_{\infty}$ nominal controller, designed to comply with the requirements of the healthy plant. Considering the shown performances, it is possible to expect that the proposed methodology acts to retard damage caused by persistent vibration. In the damage occurrence, results show the methodology effectiveness to control abnormal structural vibrations, which permit to expect the extension of the structure operational life. 


\section{Acknowledgments}

The author Helói F. G. Genari gratefully acknowledges the financial support received from CNPq ("Bolsista CNPq - Proc. no. 141621/2012-5") and CAPES ("Bolsista da CAPES - Proc. no. 12337/13-7").

\section{Appendix A. Material properties}

The material properties of the active aluminum structure are presented in Table A.4. The piezoelectric element properties are shown in Table A.5.

Table A.4

Structure mechanical properties.

\begin{tabular}{lll}
\hline Dimension $(\mathrm{mm})$ & $\rho\left(\mathrm{kg} / \mathrm{m}^{3}\right)$ & $E(\mathrm{GPa})$ \\
\hline $30 \times 600 \times 3.2$ & 2700 & 70 \\
\hline
\end{tabular}

\section{Table A.5}

Mechanical and electrical properties of the piezoelectric elements (NOLIAC ${ }^{\mathrm{ki}}$ ).

\begin{tabular}{llllll}
\hline Dimension $(\mathrm{mm})$ & $\rho\left(\mathrm{kg} / \mathrm{m}^{3}\right)$ & $E_{11}(\mathrm{GPa})$ & $E_{33}(\mathrm{GPa})$ & $d_{33}(\mathrm{pC} / \mathrm{N})$ & $d_{31}(\mathrm{pC} / \mathrm{N})$ \\
\hline $20 \times 30 \times 0.5$ & 7600 & 62.50 & 52.63 & 0.38 & -195 \\
\hline
\end{tabular}

\section{References}

[1] E.F. Crawley, J. Luis, Use of piezoelectric actuators as elements of intelligent structures, AIAA J. 25 (10) (1987) $1373-1385$.

[2] T.C. Manjunath, B. Bandyopadhyay, Fault tolerant control of flexible smart structures using robust decentralized periodic output feedback technique, Smart Mater. Struct. 14 (2005) 624-636.

[3] N. Mechbal, E.G.O. Nóbrega, Spatial $H_{\infty}$ approach to damage-tolerant active control, Struct. Control Health Monit. 22 (9) (2015) $1148-1172$.

[4] R. Isermann, Fault-Diagnosis Systems: An Introduction from Fault Detection to Fault Tolerance, Springer, 2006.

[5] C. Fendzi, N. Mechbal, M. Rébillat, M. Guskov, G. Coffignal, A general bayesian framework for ellipse-based and hyperbola-based damage localization in anisotropic composite plates, J. Intell. Mater. Syst. Struct. (2015) 1-25.

[6] C.G. Gonsalez, S. Silva, M.J. Brennan, V. Lopes Junior, Structural damage detection in an aeronautical panel using analysis of variance, Mech. Syst. Signal Process. 52-53 (2015) 206-216.

[7] W. Gawronski, Modeling and Control of Antennas and Telescopes, Springer, 2008.

[8] D.A. Pereira, A.L. Serpa, Bank of $H_{\infty}$ filters for sensor fault isolation in active controlled flexible structures, Mech. Syst. Signal Process. 60-61 (2015) 678-694.

[9] F. Ubertini, I. Venanzi, G. Comanducci, Considerations on the implementation and modeling of an active mass driver with electric torsional servomotor, Mech. Syst. Signal Process. 58-59 (2015) 53-69.

[10] L.R. Ray, L. Tian, Damage detection in smart structures through sensitivity enhancing feedback control, J. Sound Vib. 227 (5) (1999) 987-1002.

[11] Y.L. Xu, B. Chen, Integrated vibration control and health monitoring of building structures using semi-active friction dampers: part i - methodology, Eng. Struct. 30 (7) (2008) 1789-1801.

[12] B. Chen, Y.L. Xu, Integrated vibration control and health monitoring of building structures using semi-active friction dampers: part ii - numerical investigation, Eng. Struct. 30 (3) (2008) 573-587.

[13] Q. Huang, Y.L. Xu, J.C. Li, Z.Q. Su, H.J. Liu, Structural damage detection of controlled building structures using frequency response functions, J. Sound Vib. 331 (15) (2012) 3476-3492.

[14] D.J. Inman, M. Ahmadian, R.O. Claus, Simultaneous active damping and health monitoring of aircraft panels, J. Intell. Mater. Syst. Struct. 12 (11) (2001) 775-783.

[15] M.-H. Kim, Simultaneous structural health monitoring and vibration control of adaptive structures using smart materials, Shock Vib. 9 (6) (2002) 329-339.

[16] I. Ursu, G. Tecuceanu, A. Toader, V. Berar, Simultaneous active vibration control and health monitoring of structures, Exp. Results Incas Bull. 2 (2) (2010) 118-127.

[17] L. Huo, G. Song, S. Nagarajaiah, H. Li, Semi-active vibration suppression of a space truss structure using a fault tolerant controller, J. Vib. Control 18 (10) (2011) 1436-1453.

[18] K. Karami, F. Amini, Decreasing the damage in smart structures using integrated online DDA/ISMP and semi-active control, Smart Mater. Struct. 21 (10) (2012) 1-15.

[19] J. He, Q. Huang, Y.-L. Xu, Synthesis of vibration control and health monitoring of building structures under unknown excitation, Smart Mater. Struct. 23 (10) (2014) 1-13.

[20] F. Amini, S.A. Mohajeri, M. Javanbakht, Semi-active control of isolated and damaged structures using online damage detection, Smart Mater. Struct. 24 (10) (2015) 1-13.

[21] A. Preumont, Vibration Control of Active Structures: An Introduction, 3rd edition, . Springer, 2011.

[22] K. Hiramoto, T. Matsuoka, K. Sunakoda, Simultaneous optimal design of the structural model for the semi-active control design and the model-based semi-active control, Struct. Control Health Monit. 21 (4) (2014) 522-541. 
[23] Y. Zhang, J. Jiang, Bibliographical review on reconfigurable fault-tolerant control systems, Annu. Rev. Control 32 (2) (2008) $229-252$.

[24] M. Rodrigues, H. Hamdi, N.B. Braiek, D. Theilliol, Observer-based fault tolerant control design for a class of LPV descriptor systems, J. Frankl. Inst. 351 (6) (2014) 3104-3125.

[25] T. Wang, W. Xie, Y. Zhang, Sliding mode reconfigurable fault tolerant control for nonlinear aircraft systems, J. Aerosp. Eng. 28 (3) (2015) 1-11.

[26] A. Chattopadhyay, C. Nam, Y. Kim, Damage detection and vibration control of a delaminated smart composite plate, Adv. Compos. Lett. 9 (1) (2000) $7-15$.

[27] K. Umesh, R. Ganguli, Shape and vibration control of a smart composite plate with matrix cracks, Smart Mater. Struct. 18 (2) (2008) 1-13.

[28] B. Chomette, S. Chesné, D. Rémond, L. Gaudiller, Damage reduction of on-board structures using piezoelectric components and active modal control: application to a printed circuit board, Mech. Syst. Signal Process. 24 (2) (2010) 352-364.

[29] H.T.Y. Yang, J. Shan, C.J. Randall, P.K. Hansma, W. Shi, Integration of health monitoring and control of building structures during earthquakes, J. Eng. Mech. 140 (5) (2014) 1-15.

[30] C.R. Ashokkumar, Vibration control for structural damage mitigation, J. Vib. Control 21 (15) (2014) 2995-3006.

[31] P. Ambrosio, G. Cazzulani, F. Resta, F. Ripamonti, An optimal vibration control logic for minimising fatigue damage in flexible structures, J. Sound Vib. 333 (5) (2014) 1269-1280.

[32] F. Ripamonti, G. Cazzulani, S. Cinquemani, F. Resta, A. Torti, Adaptive active vibration control to improve the fatigue life of a carbon-epoxy smart structure, in: Active and Passive Smart Structures and Integrated Systems, San Diego, USA, pp. 1-9, 2015.

[33] N. Mechbal, E.G.O. Nóbrega, Adaptive Strategy to Damage-Tolerant Active Control, in: Proceedings of the 9th IFAC Symposium on Fault Detection, Supervision and Safety for Technical Processes, Paris, France, pp. 658-663, 2015.

[34] H.F.G. Genari, N. Mechbal, G. Coffignal, E.G.O. Nóbrega, Damage-Tolerant Active Control Using a Modal $H_{\infty}-$ Norm-Based Methodology, Control Engineering Practice, 2016, (in press).

[35] H.F.G. Genari, N. Mechbal, G. Coffignal, E.G.O. Nóbrega, A Modal $H_{\infty}$-Norm-based Performance Requirement for Damage-tolerant Active Controller Design, 2016, (in preparation).

[36] H.F.G. Genari, N. Mechbal, G. Coffignal, E.G.O. Nóbrega, A modal $H_{\infty}$ control methodology for damage-tolerant active control, in: Proceedings of the 9th IFAC Symposium on Fault Detection, Supervision and Safety for Technical Processes, Paris, France, pp. 664-669, 2015.

[37] J.D. Bošković, N. Knoebel, R.K. Mehra, An integrated approach to damage accommodation in flight control, in: Proceedings of the AIAA Guidance, Navigation and Control Conference and Exhibit, Honolulu, Hawaii, pp. 1-25, 2008.

[38] N. Mechbal, E.G.O. Nóbrega, Damage tolerant active control: concept and state of the art, in: Proceedings of the 8th IFAC Symposium on Fault Detection, Supervision and Safety of Technical Processes, Mexico City, Mexico, pp. 63-71, 2012.

[39] K. Zhou, Z. Ren, A new controller architecture for high performance, robust, and fault-tolerant control, IEEE Trans. Autom. Control 46 (10) (2001) $1613-1618$.

[40] J. Cieslak, D. Henry, A. Zolghadri, P. Goupil, Development of an active fault-tolerant flight control strategy, J. Guid. Control Dyn. 31 (1) (2008) 135-147.

[41] M.A.O. Alves Jr., E.G.O. Nóbrega, T. Yoneyama, Adaptive neural control for a tolerant fault system, in: Proceedings of the 7th IFAC Symposium on Fault Detection, Supervision and Safety for Technical Processes, Barcelona, Spain, pp. 137-142, 2009.

[42] J. Qiu, M. Ren, Y. Zhao, Y. Guo, Active fault-tolerant control for vehicle active suspension systems in finite-frequency domain, IET Control Theory Appl. 5 (13) (2011) 1544-1550.

[43] B. Chomette, D. Rémond, S. Chesné, L. Gaudiller, Semi-adaptive modal control of on-board electronic boards using an identification method, Smart Mater. Struct. 17 (6) (2008) 1-8.

[44] Y. Zhang, J. Jiang, Bibliographical review on reconfigurable fault-tolerant control systems, Annu. Rev. Control 32 (2) (2008) $229-252$.

[45] E. Lavretsky, K. Wise, Robust and Adaptive Control with Aerospace Applications, Springer, 2013.

[46] W. Gawronski, Advanced Structural Dynamics and Active Control of Structures, Springer, 2004.

[47] K. Zhou, J.C. Doyle, Essentials of Robust Control, Prentice Hall, 1997.

[48] S. Boyd, L. El Ghaoui, E. Feron, V. Balakrishnan, Linear Matrix Inequalities in Systems and Control Theory, SIAM, Philadelphia, 1994.

[49] C. Scherer, P. Gahinet, M. Chilali, Multiobjective output-feedback control via LMI optimization, IEEE Trans. Autom. Control 42 (7) (1997) 896-911.

[50] P. Gahinet, A. Nemirovski, A.J. Laub, M. Chilali, LMI control toolbox: for use with MATLAB ${ }^{15}$, MathWorks (1995).

[51] H. Hamdi, M. Rodrigues, C. Mechmeche, N.B. Braiek, Robust fault detection and estimation for descriptor systems based on multi-models concept, Int. J. Control Autom. Syst. 10 (6) (2012) 1260-1266.

[52] F. Casciati, G. Magonette, F. Marazzi, Technology of Semiactive Devices and Applications in Vibration Mitigation, Wiley, 2006.

[53] K. Zhou, J.C. Doyle, K. Glover, Robust and Optimal Control, Prentice Hall, 1995.

[54] J.L. Batoz, K.J. Bathe, L.W. Ho, A study of three-node triangular plate bending elements, Int. J. Numer. Methods Eng. 15 (12) (1980) 1771-1812.

[55] A. Preumont, Vibration Control of Active Structures: An Introduction, Kluwer Academic Publishers, 2002. 